\title{
Demonstration of hetero-gate-dielectric tunneling field-effect transistors (HG TFETs)
}

\author{
Woo Young Choi* and Hyun Kook Lee
}

\begin{abstract}
The steady scaling-down of semiconductor device for improving performance has been the most important issue among researchers. Recently, as low-power consumption becomes one of the most important requirements, there have been many researches about novel devices for low-power consumption. Though scaling supply voltage is the most effective way for low-power consumption, performance degradation is occurred for metal-oxide-semiconductor field-effect transistors (MOSFETs) when supply voltage is reduced because subthreshold swing (SS) of MOSFETs cannot be lower than $60 \mathrm{mV} / \mathrm{dec}$. Thus, in this thesis, hetero-gate-dielectric tunneling field-effect transistors (HG TFETs) are investigated as one of the most promising alternatives to MOSFETs. By replacing source-side gate insulator with a high-k material, HG TFETs show higher on-current, suppressed ambipolar current and lower SS than conventional TFETs. Device design optimization through simulation was performed and fabrication based on simulation demonstrated that performance of HG TFETs were better than that of conventional TFETs. Especially, enlargement of gate insulator thickness while etching gate insulator at the source side was improved by introducing HF vapor etch process. In addition, the proposed HG TFETs showed higher performance than our previous results by changing structure of sidewall spacer by high-k etching process.
\end{abstract}

Keywords: Tunneling field-effect transistors (TFETs), Metal-oxide-semiconductor field-effect transistors (MOSFETS)

\section{Background}

The steady scaling-down of semiconductor device with rapid progress of fabrication technology facilitated high-integration, high-performance [1]. However, scaling-down resulted in short channel effects and power consumption increased exponentially $[2,3]$. Recently, low power consumption becomes one of the most important requirements as scaling-down in semiconductor industry with the rapid growth of mobile market.

The most efficient way to reduce power consumption is to scaling supply voltage $\left(V_{\mathrm{DD}}\right)$ down which plays an important role in determining both standby and dynamic power consumptions. However, $V_{\mathrm{DD}}$ scaling of MOSFETs has been slower than device scaling because the downscaling of threshold voltage $\left(V_{\mathrm{T}}\right)$ leads to a dramatic increase of off-current $\left(I_{\text {off }}\right)$ as described in Fig. 1 [4]. This is closely related to fundamental limit that subthreshold

*Correspondence: wchoi@sogang.ac.kr Department of Electronic Engineering, Sogang University, Seoul 04107, Republic of Korea swing (SS) of MOSFETs cannot be lower than $60 \mathrm{mV} / \mathrm{dec}$ [5]. In the case of MOSFETs, carriers are injected from the source to the channel by thermionic emission mechanism. As the energy distribution of conduction electrons in the source follows the Fermi-Dirac distribution, electrons injected by increasing gate voltage $\left(V_{\mathrm{G}}\right)$ also follow the Fermi-Dirac distribution which limits minimal SS around $60 \mathrm{mV} / \mathrm{dec}$ at room temperature.

Thus, many novel devices have been proposed recently to overcome fundamental limit. They include impactionization MOS devices [5, 6], nano-electro-mechanical FETs [7], and tunneling field-effect transistor (TFET) [823]. Among them, a TFET is considered one of the most promising candidates for ultra-low-power semiconductor device. TFETs show low $I_{\text {off }}$ and sub- $60 \mathrm{mV} / \mathrm{dec} S \mathrm{~S}$ at room temperature because electron flows are controlled by band-to-band tunneling mechanism. In addition, TFETs are less influenced by short channel effects than MOSFETs $[14,15]$ and complementary metal-oxide semiconductor (CMOS) process compatible. On the other hand, TFETs have disadvantages such as lower on current 


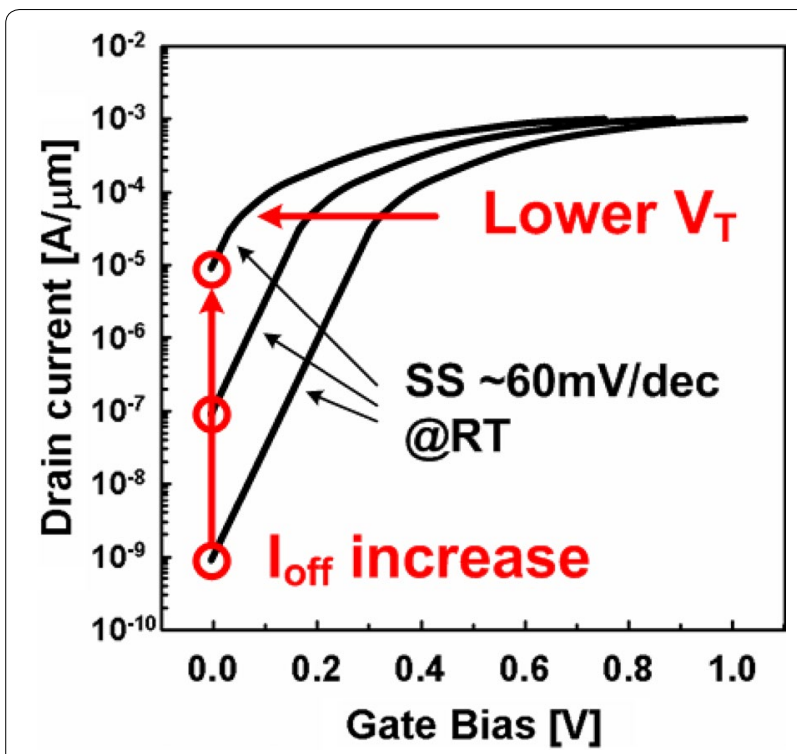

Fig. 1 Threshold voltage scaling problem

$\left(I_{\text {on }}\right)$ and ambipolar behavior $[16,17]$. To overcome these problems, many studies have been reported by introducing various materials and device structures [17-23].

In this thesis, hetero-gate-dielectric tunneling fieldeffect transistors (HG TFETs) are investigated. HG TFETs show higher $I_{\text {on }}$, lower ambipolar current $\left(I_{\mathrm{amb}}\right)$ and smaller SS than conventional TFETs by replacing sourceside gate insulator with high- $k$ materials [18]. First, the theoretical background of TFETs and device concepts of HG TFETs will be covered. In addition, HG TFET design was optimized and improved through the simulation. As a result, HG TFETs showed higher performance than that of conventional TFETs. To improve the performance of HG TFETs, improved fabrication methods were proposed. Etching the gate insulator at the source side by using HF vapor improved enlargement of etched gate insulator thickness. In addition, structure of sidewall spacers was changed to remove the high- $k$ layer on the source region by high- $k$ etching process. This solved the problem that tunneling barrier width was increased by fringe field. After the overall process flow for the fabricating HG TFETs using standard CMOS process was introduced, electrical characteristic results of fabricated device demonstrated the simulation results. Proposed HG TFETs showed higher performance than our previous results. As a result, it is concluded that HG TFETs are promising to be used for highly energy efficient ICs.

\section{Theoretical studies}

\subsection{Basic operations of TFETs}

Compared to MOSFETs, basic structure of TFETs is a gated $\mathrm{p}-\mathrm{i}-\mathrm{n}$ diode as shown in Fig. 2. Band-to-band tunneling mechanism is used as a carrier injection of TFETs instead of thermionic emission. Different operation mechanism between MOSFETs and TFETs comes from the asymmetric doping profile of source and drain of TFETs. In $\mathrm{n}$-channel TFETs, the $\mathrm{p}^{+}$source is grounded and the $\mathrm{n}^{+}$drain is positively biased. In the off-state, TFETs resemble a reverse biased $\mathrm{p}-\mathrm{i}-\mathrm{n}$ diode and tunneling barrier width $\left(W_{\text {tun }}\right)$ between valence band of the source and conduction band of the channel is thick which make extremely low $I_{\text {off }}$ flow. In the case of MOSFETs, electron injection from the source to the channel is hard because of high energy barrier between the source and the channel. In the on-state, when a positive gate bias induces strong band bending of channel and $W_{\text {tun }}$ is narrowed, the valence band electrons from the source region tunnel through the barrier into the conduction band in the channel region. Thus, the TFET shows very sharp on-off transition and SS value of TFETs is not subjected to $60 \mathrm{mV} / \mathrm{dec}$ thermal limit like MOSFETs. These characteristics lead a TFET as one of the most promising candidates for low-power device. Despite those advantages, TFETs have several disadvantages to figure out. Because of high tunneling resistance, $I_{\text {on }}$ of TFETs is much lower than that of MOSFETs and ambipolar behavior of TFETs increases leakage current [8]. To improve performance of TFETs, various techniques have been proposed. Since $I_{\text {on }}$ of TFETs is determined by $W_{\text {tun }}$ and electric field at the tunneling junction, introducing high- $k$ materials as a gate insulator, narrow bandgap materials and novel device structures were shown. However, using high $-k$ materials as a gate insulator increases $I_{\text {amb }}$ by ambipolar behavior as well as $I_{\text {on }}$ [14].

\subsection{Characteristics of HG TFETs}

HG TFETs are proposed for higher $I_{\text {on }}$, lower $I_{\text {amb }}$, and smaller SS. In this study, HG TFETs will be compared with two kinds of conventional TFETs, high- $k$-only and $\mathrm{SiO}_{2}-$ only TFETs as shown in Fig. 3. High- $k$-only TFETs use only high- $k$ dielectric as gate insulator and $\mathrm{SiO}_{2}$-only TFETs use only silicon oxide $\left(\mathrm{SiO}_{2}\right)$ as a gate insulator. The HG TFET is composed of different gate dielectric materials at the source and drain sides. A high- $k$ material is only partially located at the source side and this leads to the particular energy band structure as shown in Fig. 4. HG TFETs show a local minimum of the conduction band edge $\left(E_{\mathrm{c}}\right)$ due to relative permittivity discrepancy between high- $k$ dielectric and $\mathrm{SiO}_{2}$ layer. HG TFETs show more abrupt change from off to on-state because $W_{\text {tun }}$ of HG TFETs abruptly narrows when a local minimum of $E_{\mathrm{c}}$ is aligned with the valence band edge $\left(E_{\mathrm{v}}\right)$ of the source region.

To compare the performance of HG TFETs with high$k$-only and $\mathrm{SiO}_{2}$-only TFETs, two-dimensional device simulation has been performed by using Silvaco ATLAS [24]. A nonlocal band-to-band tunneling model has been 


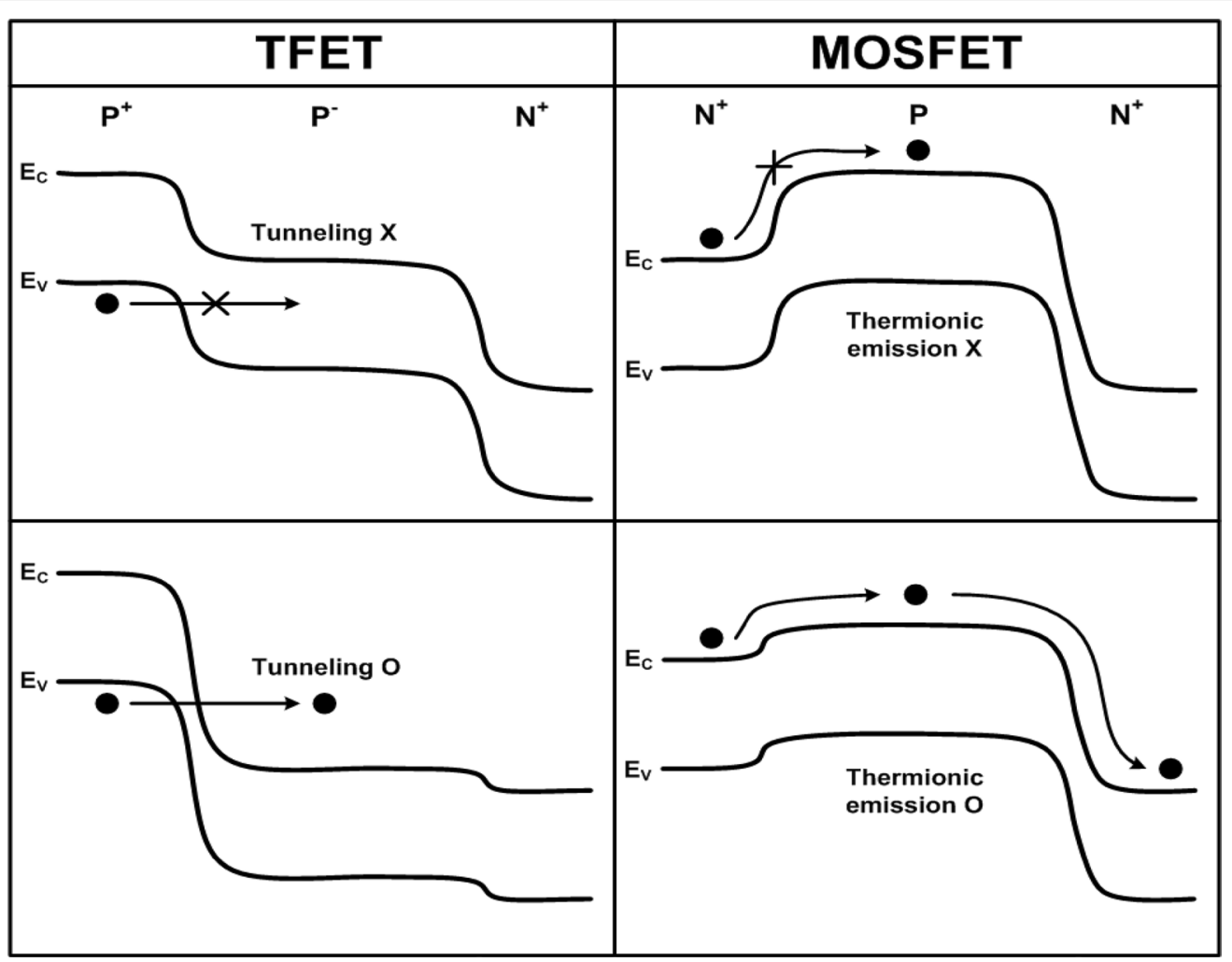

Fig. 2 Energy bands of the TFET and the MOSFET during operation

used. Band gap narrowing, Fermi statistics, ShockleyRead-Hall (SRH) recombination and Lombardi mobility models have been used in this simulation. Gate leakage current and quantum effect have been ignored. An abrupt source/drain junction profile has been assumed as shown in the previous works $[18,25]$. Device parameters used in this simulation are summarized in Table 1. Figure 5a shows the transfer characteristics of HG, high- $k$-only and $\mathrm{SiO}_{2}$-only TEFTs that use $\mathrm{n}$-type doped polysilicon gates. Optimized HG TFETs whose length of high- $k$ material under the gate $\left(L_{\text {high-k }}\right)$ is $5 \mathrm{~nm}$ are used in this case. HG TFETs follows $\mathrm{SiO}_{2}$-only TFETs at low $V_{\mathrm{G}}$ because ambipolar behavior is determined by the drain-to-channel region overlapped by $\mathrm{SiO}_{2}$ layer. On the other hand, onstate of HG TFETs follow high- $k$-only TFETs because of high- $k$ insulator locate at the source-to-channel region. For fair comparison, the gate workfunction is adjusted that $I_{\text {off }}$ is $0.1 \mathrm{fA}$ at $0 \mathrm{~V} V_{\mathrm{G}}$ as shown in Fig. 5b. Because HG TFETs show higher $I_{\text {on }}$ than high- $k$-only TFETs and have $I_{\text {amb }}$ as low as $\mathrm{SiO}_{2}$-only TFETs, HG TFETs show lower SS than high- $k$-only and $\mathrm{SiO}_{2}$-only TFETs.

\subsection{Optimization of the device design}

To optimize the device design of HG TFETs, the design issues of HG TFETs such as $L_{\text {high- } k}$ and silicon-on-insulator (SOI) layer thickness $\left(T_{\mathrm{SOI}}\right)$ have been investigated. $I_{\text {on }}$ is defined as drain current $\left(I_{\mathrm{D}}\right)$ when both $V_{\mathrm{G}}$ and drain voltage $\left(V_{\mathrm{D}}\right)$ are $0.7 \mathrm{~V}, I_{\mathrm{amb}}$ is defined as $I_{\mathrm{D}}$ when $V_{\mathrm{G}}$ is $-0.7 \mathrm{~V}$ and $V_{\mathrm{D}}$ is $0.7 \mathrm{~V}$. SS is defined as an average slope when $I_{\mathrm{D}}$ is from $0.1 \mathrm{fA} / \mu \mathrm{m}$ to $0.1 \mathrm{nA} / \mu \mathrm{m}$ at $V_{\mathrm{D}}$ is $0.7 \mathrm{~V}$. Figure $6 \mathrm{a}$ shows extracted $I_{\text {on }}$ and SS as a function of $L_{\text {high- } k}$. When $L_{\text {high- } k}$ is optimized around $5 \mathrm{~nm}$, HG TFET show $40 \%$ smaller SS and three times higher $I_{\text {on }}$ than high- $k$-only TFETs. In addition, HG TFETs show $~ 70 \%$ smaller SS and three orders of magnitude higher $I_{\text {on }}$ than $\mathrm{SiO}_{2}$-only TFETs. Figure $6 \mathrm{~b}$ shows extracted $I_{\mathrm{amb}}$ as a function of $L_{\mathrm{high}-k}$. Because $I_{\mathrm{amb}}$ is determined by ambipolar behavior at the drain side, $I_{\text {amb }}$ abruptly decrease as $L_{\text {high- } k}$ decreases. As a result, HG TFETs show six orders lower $I_{\mathrm{amb}}$ compared to high$k$-only TFETs.

In addition, the effect of $T_{\mathrm{SOI}}$ has been discussed in terms of $I_{\text {on }}$ and SS. Figure 7 shows extracted $I_{\text {on }}$ and SS as a function of $T_{\mathrm{SOI}}$ for several different operating voltage $\left(V_{\mathrm{DD}}\right)$. $I_{\text {on }}$ is defined as $I_{\mathrm{D}}$ when both $V_{\mathrm{G}}$ and $V_{\mathrm{D}}$ are equal to $V_{\mathrm{DD}}$. SS is defined as same as before. $I_{\text {on }}$ of HG TFETs show little change as $T_{\mathrm{SOI}}$ decreasing when $V_{\mathrm{DD}}$ is $0.7 \mathrm{~V}$. However, $I_{\text {on }}$ of HG TFETs tends to become lower as $T_{\text {SOI }}$ decreases at low $V_{\mathrm{DD}}$ as shown in Fig. 7b, c. In addition, decreasing $T_{\mathrm{SOI}}$ makes the SS of HG TFETs larger regardless of $V_{\mathrm{DD}}$. It 

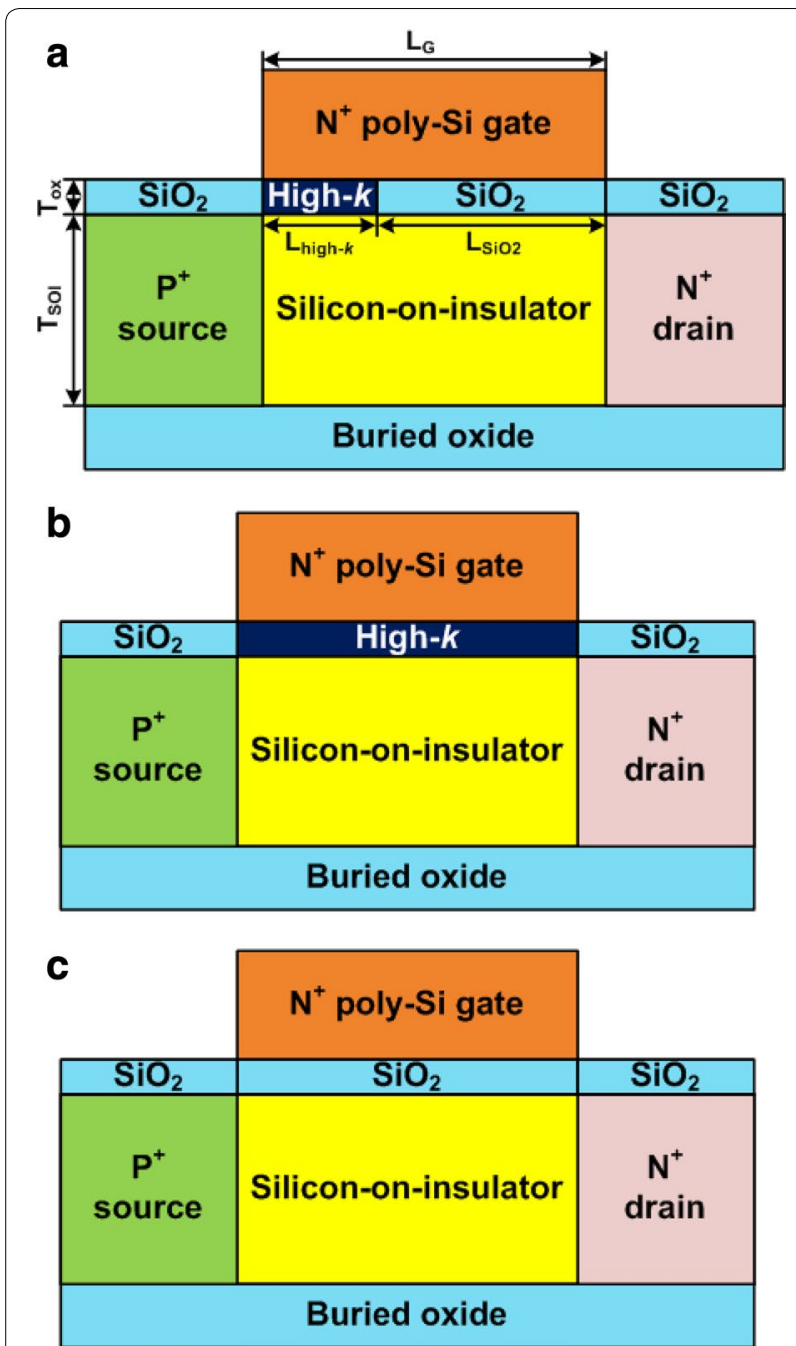

Fig. 3 Schematics of an a $\mathrm{HG}$, b high-k-only and $\mathbf{c} \mathrm{SiO}_{2}$-only TFET

is because the performance of HG TFETs is mainly determined by the difference in the gate-to-channel coupling strength between channel regions overlapped by the high- $k$ insulator and $\mathrm{SiO}_{2}$ layer and it decreases as $T_{\mathrm{SOI}}$ decreases. As a result, it is difficult to form a local minimum on the conduction band edge and performance of HG TFETs worsens as $T_{\text {SOI }}$ decreases. To sum up, large $T_{\mathrm{SOI}}$ can be helpful to get higher $I_{\mathrm{on}}$ of HG TFETs at low $V_{\mathrm{DD}}$ and SS of HG TFETs increases as $T_{\mathrm{SOI}}$ decreases regardless of $V_{\mathrm{DD}}$ [26]. As a result, $30-\mathrm{nm} T_{\mathrm{SOI}}$ is selected for fabrication this time.

\subsection{Improvement in device design}

Our previous work showed worse HG TFET performance than expected [27]. It was concluded that this result came from some factors: gradual doping profile, enlarged high$k$ dielectric thickness at the source side and sidewall spacer structures. All of these factors are related to the
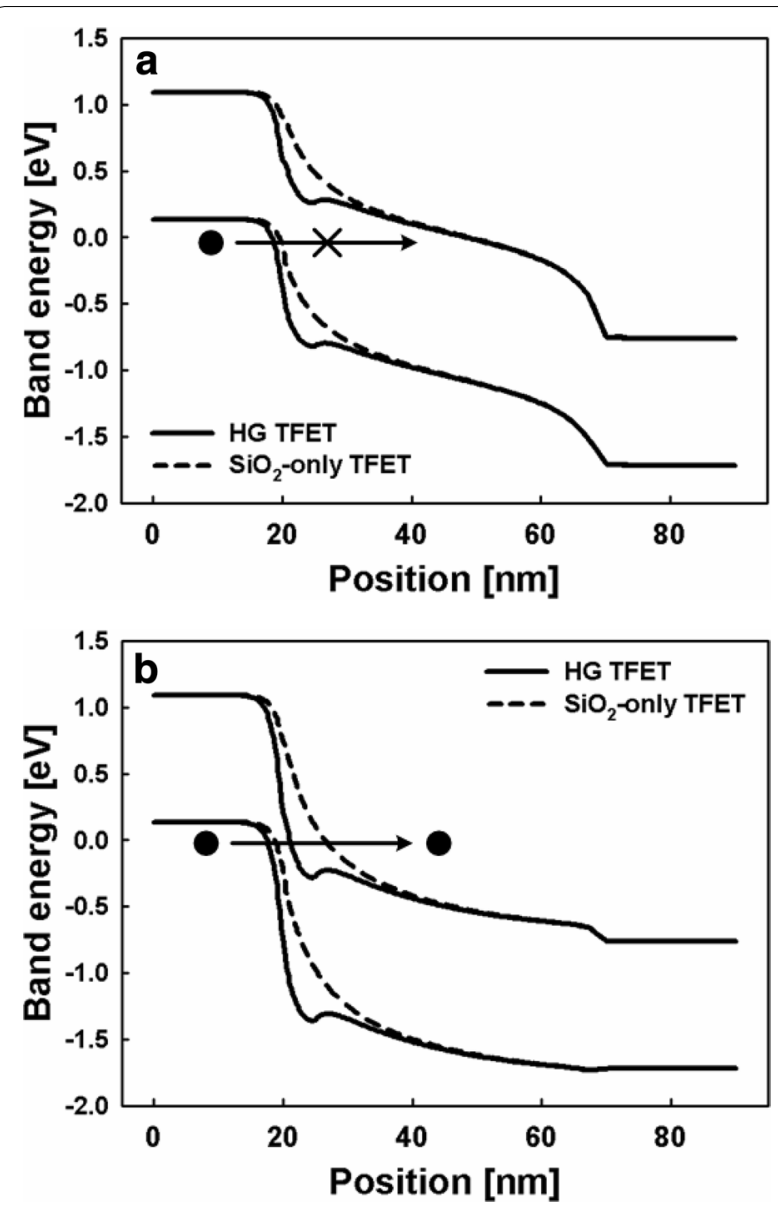

Fig. 4 Energy band diagrams of $\mathrm{HG}$ and $\mathrm{SiO}_{2}$-only TFETs at $\mathbf{a}$ off- and b on-state

Table 1 Device parameters used for simulation

\begin{tabular}{|c|c|c|c|}
\hline & HG TFET & High-k-only TFET & $\mathrm{SiO}_{2}$-only TFET \\
\hline$L_{G}(n m)$ & 50 & 50 & 50 \\
\hline$t_{\mathrm{SOI}}(\mathrm{nm})$ & 30 & 30 & 30 \\
\hline$t_{\text {ins }}(n m)$ & 2 & 2 & 2 \\
\hline $\begin{array}{l}\text { Source/drain doping } \\
\text { conc. }\left(\mathrm{cm}^{-3}\right)\end{array}$ & $10^{20}$ & $10^{20}$ & $10^{20}$ \\
\hline $\begin{array}{l}\text { Channel doping conc. } \\
\left(\mathrm{cm}^{-3}\right)\end{array}$ & $10^{15}$ & $10^{15}$ & $10^{15}$ \\
\hline$L_{\text {high-k }}(n m)$ & 5 & 50 & $x$ \\
\hline $\begin{array}{l}k \text { value of high- } k \\
\text { dielectric }\end{array}$ & 25 & 25 & $x$ \\
\hline
\end{tabular}

fabrication process and these have been investigated to improve the performance of HG TFETs.

First, abrupt doping profile at the tunneling junction is very important for TFETs because it determines $W_{\text {tun }}$ and electric field which control the tunneling current. Doping 

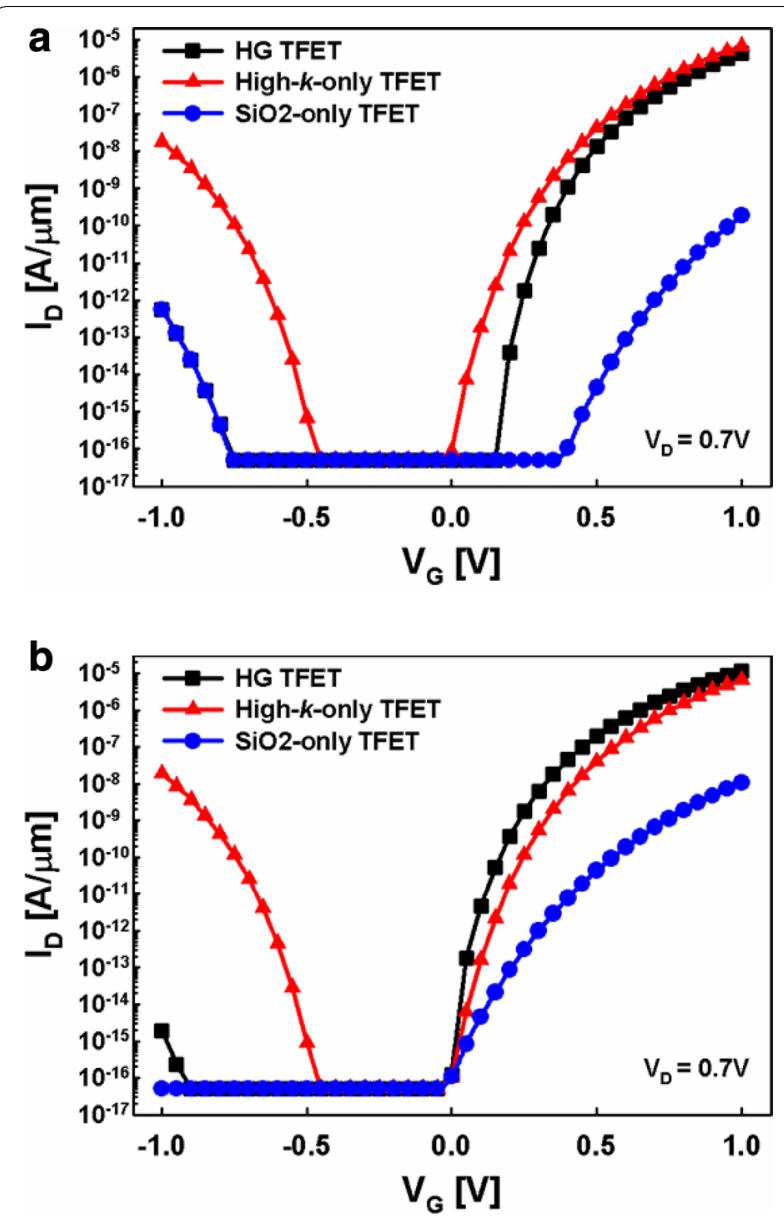

Fig. 5 Transfer curves of the $\mathrm{HG}$, high-k-only, and $\mathrm{SiO}_{2}$-only TFETs when $\mathbf{a}$ gate workfunction is $4.1 \mathrm{eV}$ and $\mathbf{b}$ gate work function is adjusted that $I_{\text {off }}$ is $0.1 \mathrm{fA}$ at $0 \mathrm{~V} V_{G}$

profile which is especially overlapped by high- $k$ material has an influence on HG TFETs because performance of HG TFETs is mostly determined by formation of a local minimum of the $E_{\mathrm{c}}$ at the tunneling junction [18]. As a result, abrupt doping profiles at the tunneling junction are suitable for higher $I_{\text {on }}$ and lower SS. However, gradual doping profiles are applied to our HG TFETs because we used conventional RTA instead of advanced annealing method. Thus, fabrication conditions which control the doping profile should be optimized. In general, doping profiles at the tunneling junction are influenced by the spacer length $\left(L_{\text {spacer }}\right)$ and the RTA time $\left(T_{\mathrm{RTA}}\right) \cdot L_{\text {spacer }}$ is the sum of an inner high- $k$ spacer length and an outer low- $k$ spacer length. To adopt the fabrication conditions, two-dimensional semiconductor process simulation and device simulation has been performed by using Silvaco ATHENA and ATLAS [24]. In the case of process simulation, some conditions were changed from the conditions used for device simulation. Abrupt doping profile
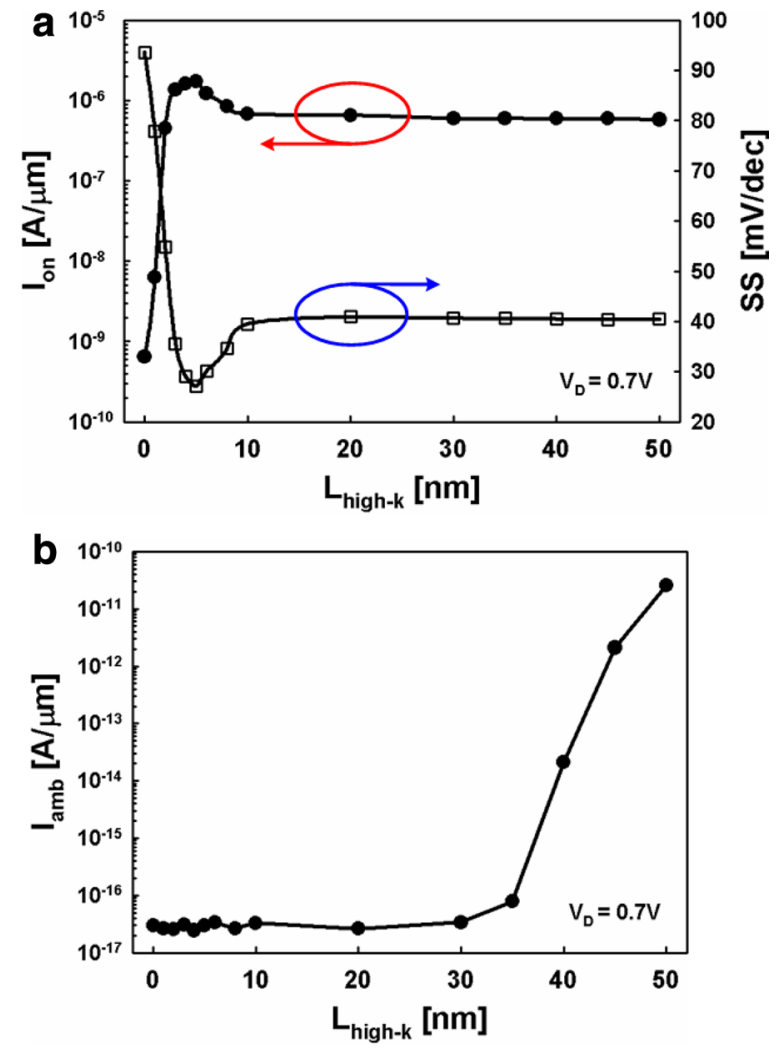

Fig. $\mathbf{6} \mathbf{a} I_{\text {on }}$ and $S S$ and $\left.\mathbf{b}\right|_{\mathrm{amb}}$ of HG TFETs as a function of $L_{\text {high-k }}$

is changed to gradual doping profile which is determined by $T_{\mathrm{RTA}}$.

Second, high- $k$ dielectric partially located at the source side increase the gate-to-channel coupling strength and this leads to the particular energy band structure [18]. HG TFETs show lower SS and higher $I_{\text {on }}$ because of a local minimum of the $E_{\mathrm{c}}$ at the tunneling region. Though the thickness of high- $k$ dielectric should be equal to $T_{\mathrm{ox}}$, this is enlarged during etch process of $\mathrm{SiO}_{2}$ gate insulator. Thus, the difference of the gate-to-channel coupling strength between channel regions overlapped by the high- $k$ dielectric and $\mathrm{SiO}_{2}$ decreased. It degrades the performance of HG TFETs and solution to this will be discussed in chapter 3.

Third, the sidewall spacer structure of our previous HG TFETs is problematic. Figure 8a shows the structure of our previous HG TFETs. Previous HG TFETs have gradual doping profiles and dual- $k$ spacers which consist of 3-nm inner high- $k$ spacers and $19-n m$ outer low- $k$ spacers. High $-k$ spacers are used to enhance the electric field around the tunneling junction and low- $k$ spacers are used to control tunneling junctions [28, 29]. However, $3-n m$ high- $k$ dielectric layers under the low- $k$ spacers are the main factors which degrade the performance of HG 

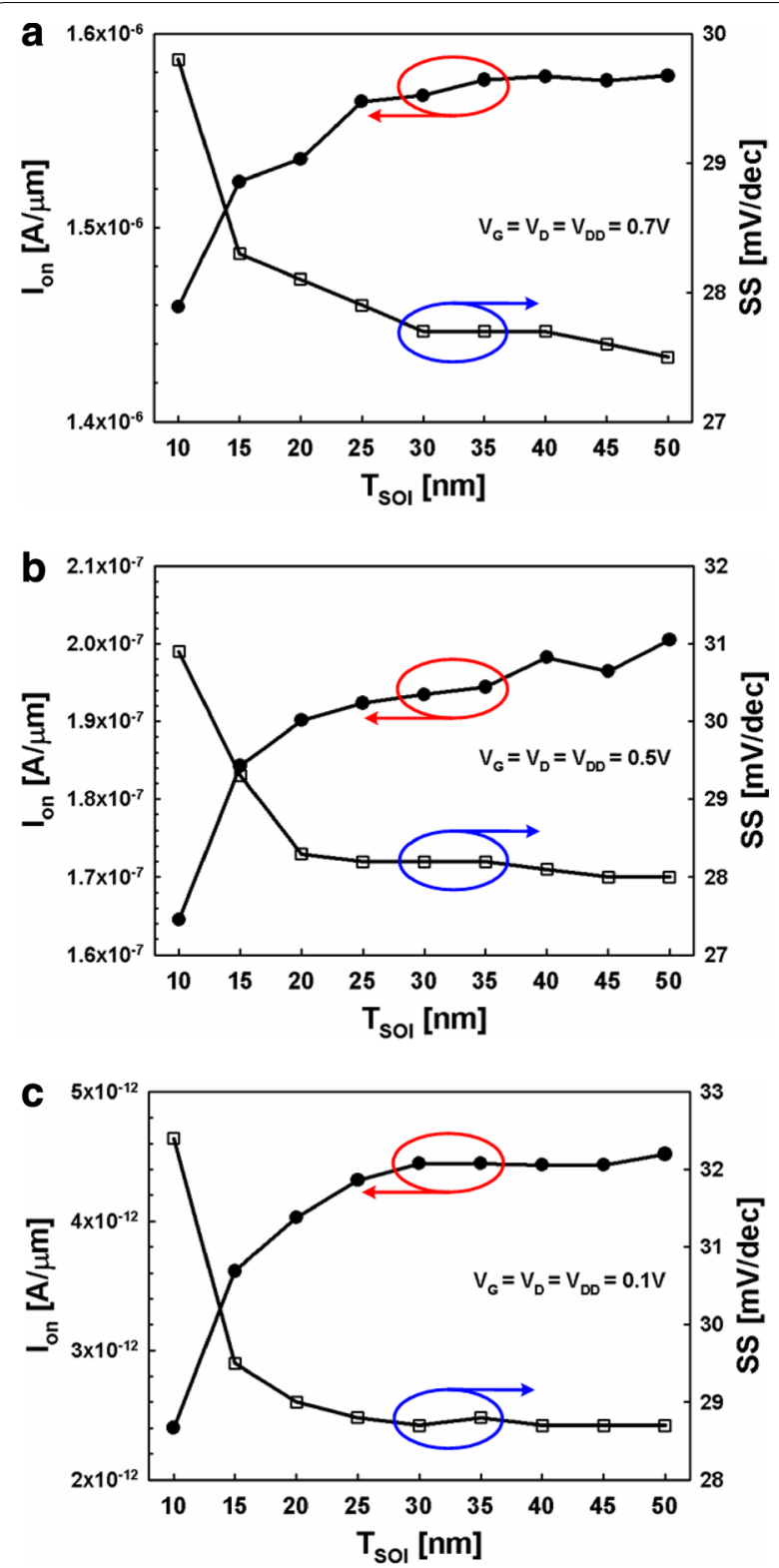

Fig. $7 I_{\text {on }}$ and SS of HG TFETS with the variation of $T_{\text {SOI }}$ for different $V_{\mathrm{DD}}$ conditions. a $V_{\mathrm{DD}}=0.7 \mathrm{~V}, \mathbf{b} V_{\mathrm{DD}}=0.5 \mathrm{~V}, \mathbf{c} V_{\mathrm{DD}}=0.1 \mathrm{~V}$

TFETs. Because high- $k$ dielectric layers are placed on the source regions, fringe field from gates increases as $V_{\mathrm{G}}$ increases. Accordingly, the energy bands of the source regions decrease as well as those of the channel regions. It makes $W_{\text {tun }}$ larger and our previous HG TFET performance worse.

To enhance the performance of HG TFETs, the dependency of sidewall spacer structures on the performance has been examined. The structure of a dual- $k$ spacer is improved as shown in Fig. 8b. A 3-nm high- $k$ dielectric layer under the low- $k$ spacer is removed and only a 3-nm

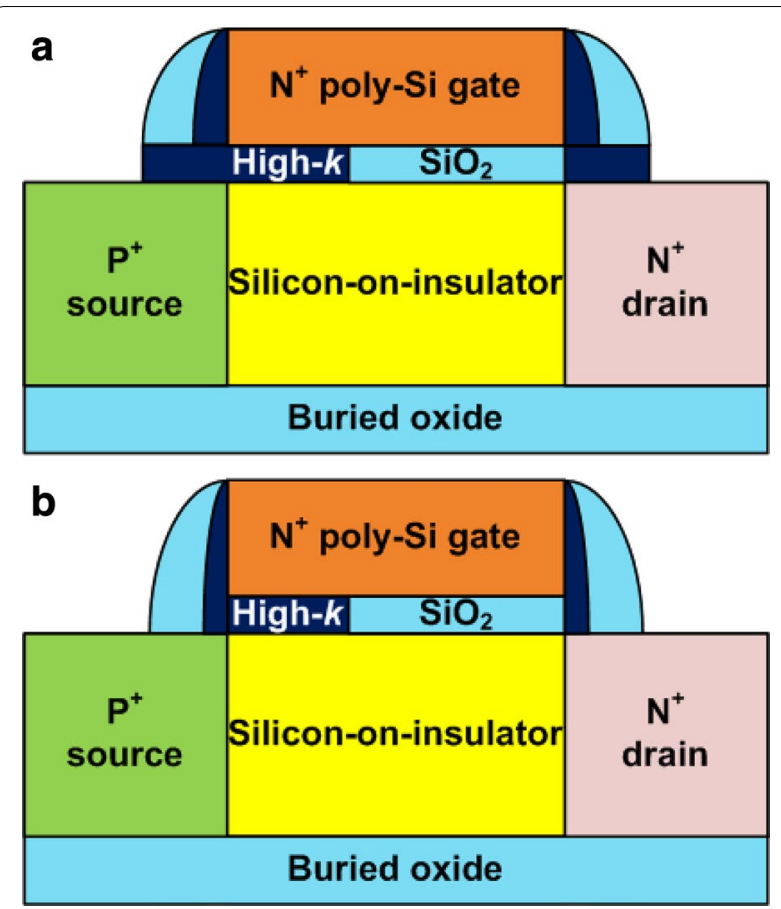

Fig. 8 Structures of an a previous and $\mathbf{b}$ proposed HG TFET

inner high- $k$ spacer is remained. To investigate the impact of the dual- $k$ spacer structure on the performance of $\mathrm{HG}$ TFETs, fringe field around the tunneling region is compared as shown in Fig. 9. Inner high- $k$ spacer increases the fringe field around the tunneling junction for both structures. Fringe field coupling through the inner high$k$ spacer decreases $W_{\text {tun }}$ [28]. However, fringe fields are denser and higher near the junction in the proposed HG TFETs compared to the previous HG TFETs. In the case of proposed HG TFETs, fringe field is focused on the edge of the high- $k$ spacer which is in contact with TEOS spacer. On the other hand, fringe field of the previous HG TFETs is low and spread because fringe field through the inner high $-k$ spacer and high $-k$ dielectric layer under the low- $k$ spacer are balanced. Thus, gate potential is coupled over a large distance and this result in low current.

The impact of the fringe field coupling on the tunneling region is further illustrated by the band diagrams as shown in Fig. 10. The figure shows the band diagrams near the tunneling junction for $V_{\mathrm{G}}=V_{\mathrm{D}}=0.7 \mathrm{~V}$. From the figure, $W_{\text {tun }}$ of the previous and proposed HG TFETs have been compared each other. As mentioned before, $W_{\text {tun }}$ of the previous HG TFETs is larger than that of proposed HG TFETs because fringe field through the high$k$ dielectric layer on the source region reduce the energy band of the source region.

To optimize the design of HG TFETs, the effect of variation in the length of the high- $k$ spacer on $I_{\text {on }}$ has been 

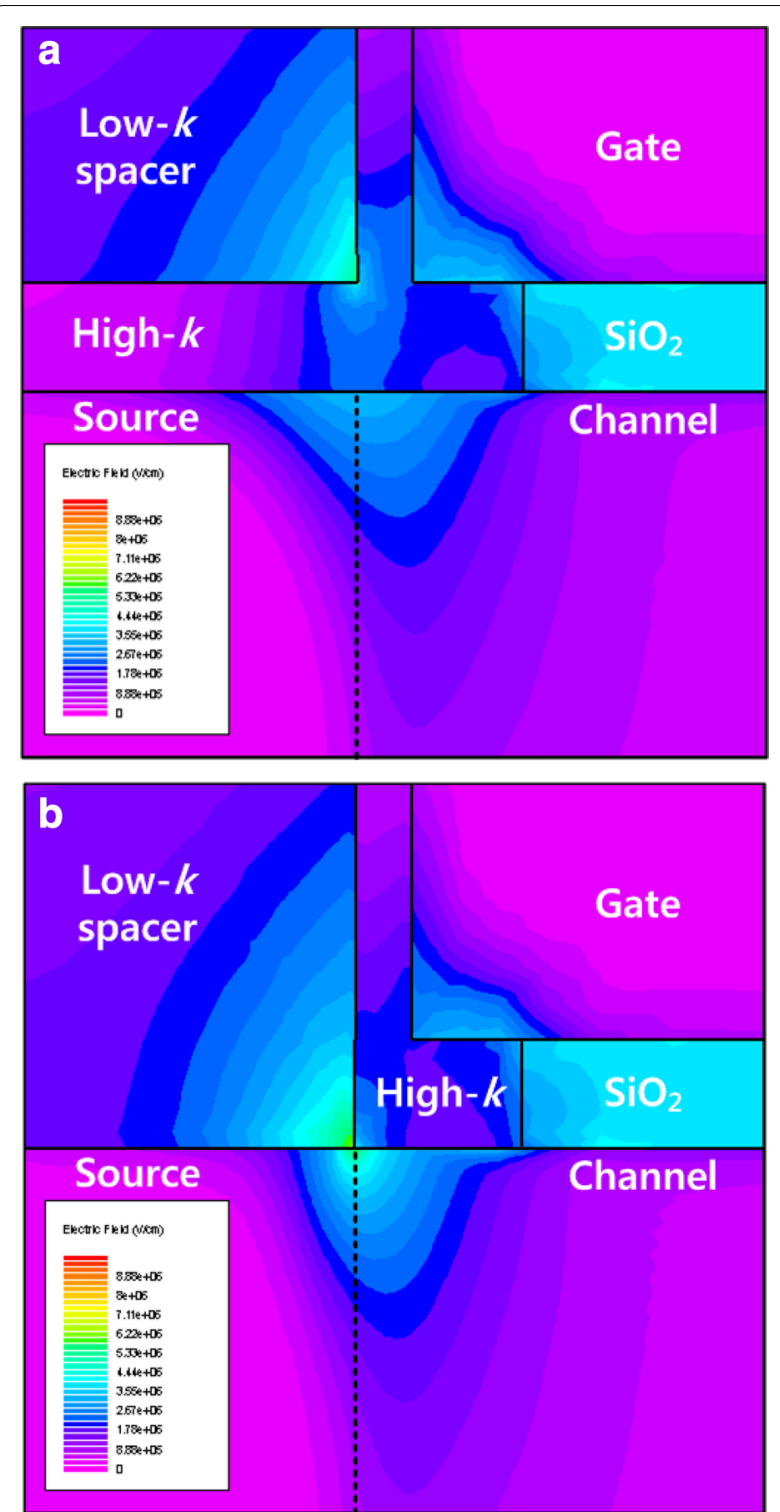

Fig. 9 Fringe field through the spacer for $\mathbf{a}$ previous and $\mathbf{b}$ proposed HG TFETS

investigated. Figure 11 shows the transfer characteristics of the proposed HG TFETs compared with the previous HG TFETs as the length of the high- $k$ spacer varies from 0 to $5 \mathrm{~nm}$. The length of an outer low- $k$ spacer is fixed at $19 \mathrm{~nm}$ for all because of the tunneling junction. Performance degradation is more severe in the case of the previous HG TFETs because high- $k$ dielectric on the source region increases the coupling between the gate and the source region. It is clear from the transfer characteristics in Fig. 11 that the device performance degrades with an increasing the length of the high- $k$ spacer for the proposed HG TFETs. An increase in the length of the high- $k$

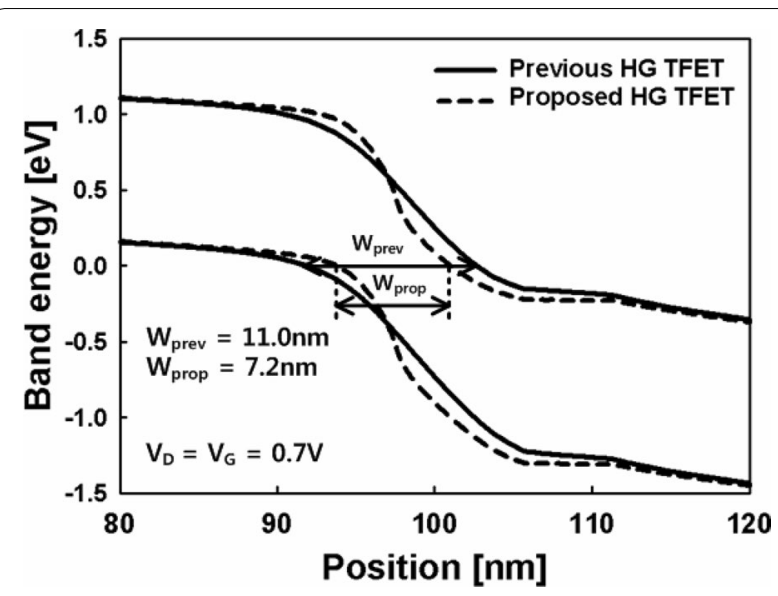

Fig. 10 Energy band diagrams for the previous and proposed HG TFETs. $W_{\text {prev }}$ and $W_{\text {prop }}$ refer to the tunneling width for the previous and proposed HG TFETs

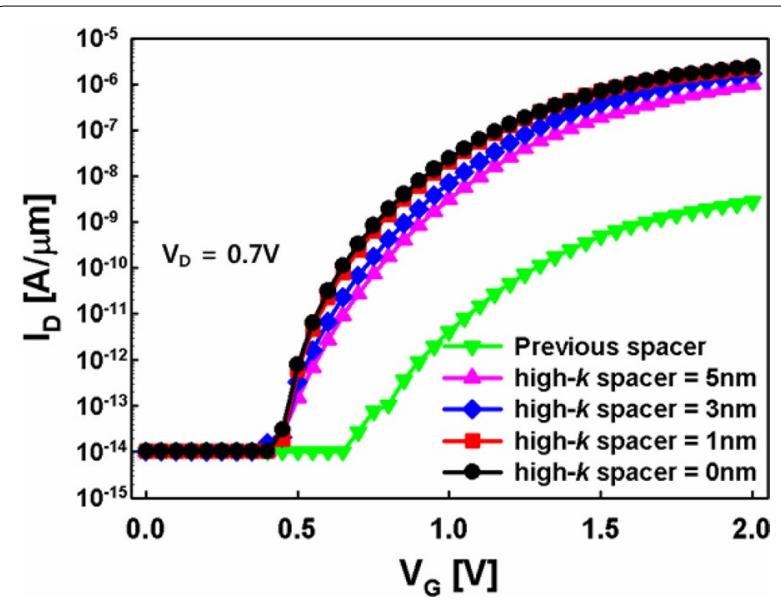

Fig. 11 Effect of high-k spacer length variation on the transfer characteristics for the proposed HG TFETs which is compared to the previous HG TFETs

spacer reduces the electric field from the gate because of the physical distance, thereby causing the degradation in the device performance.

Figure 12 shows the energy band diagrams of the proposed HG TFETs with various high- $k$ spacer lengths at $V_{\mathrm{G}}=V_{\mathrm{D}}=0.7 \mathrm{~V}$. $W_{\text {tun }}$ was extracted from the point which shows the maximum electron tunneling rate. $W_{\text {tun }}$ increases as the length of the high- $k$ spacer increases which is consistent with the trend in the transfer characteristics. As a result, HG TFETs without an inner high- $k$ spacer show the most improved performance. However, the length of high- $k$ spacer in this study is $3 \mathrm{~nm}$ because of the fabrication issues and this will be covered in chapter 3. 


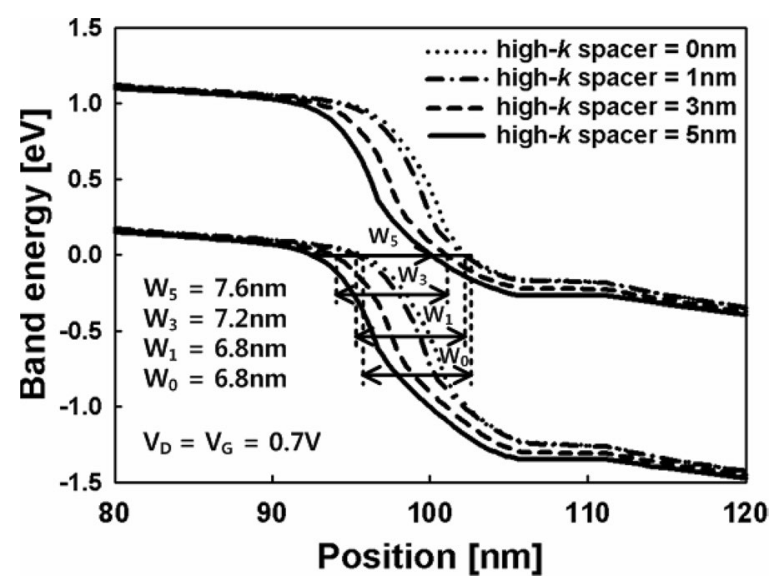

Fig. 12 Energy band diagrams for proposed HG TFETs with the variation of high- $k$ spacer lengths

To verify the fabrication condition for the proposed HG TFETs, the effects of $L_{\text {spacer }}$ and $T_{\text {RTA }}$ have been also discussed in terms of $I_{\text {on }}$ and SS. Figure 13a shows extracted SS as a function of $L_{\text {spacer }}$ and $T_{\text {RTA. When the device }}$ structure is formed by process simulation, SS is extracted from different range of $I_{\mathrm{D}}$ because leakage current level and average of SS are higher than those of device simulation. Thus, SS is defined as an average slope when $I_{\mathrm{D}}$ increases from 10 to $100 \mathrm{fA} / \mu \mathrm{m}$. Regardless of $T_{\mathrm{RTA}}$, SS of HG TFETs becomes higher as $L_{\text {spacer }}$ decreases because dopants of high concentration diffused from the source region are overlapped by high- $k$ material. In this case, conduction band well becomes shallower because higher doping concentration makes $E_{\mathrm{c}}$ under the high- $k$ material increases. On the other hands, SS becomes higher as $L_{\text {spacer }}$ increases because of underlap between source and channel region. Similarly, when $L_{\text {spacer }}$ is fixed, SS becomes higher as $T_{\mathrm{RTA}}$ decreases because of underlap structure. On the contrary, as $T_{\mathrm{RTA}}$ increases, conduction band well becomes shallower, which makes less abrupt transition between off- and on-state. When $T_{\text {RTA }}$ is $3 \mathrm{~s}$, minimum SS value is shown when $L_{\text {spacer }}$ is $24 \mathrm{~nm}$ and optimum $L_{\text {spacer }}$ increases as $T_{\text {RTA }}$ increases.

Figure $13 \mathrm{~b}$ shows extracted $I_{\text {on }}$ as a function of $L_{\text {spacer }}$ and $T_{\mathrm{RTA}}$. The turn-on voltage $\left(V_{\text {turn-on }}\right)$ is defined as $V_{\mathrm{G}}$ when $I_{\mathrm{D}}$ is $10 \mathrm{fA} / \mu \mathrm{m}$. $I_{\text {on }}$ is defined as $I_{\mathrm{D}}$ when $V_{\mathrm{D}}$ is $0.7 \mathrm{~V}$ and $V_{\mathrm{G}}$ is $0.7 \mathrm{~V}$ higher than $V_{\text {turn-on. }} I_{\text {on }}$ shows similar tendency observed in SS as a function of $L_{\text {spacer }}$ and $T_{\text {RTA }}$. Optimum $L_{\text {spacer }}$ increases as $T_{\text {RTA }}$ increases for the same reason. Tunneling current increases as electric field at the tunneling region increases and it is reversely exponential to $W_{\text {tun }}$. Electric field is determined by the slope of the energy level in the band diagrams and $W_{\text {tun }}$ is also
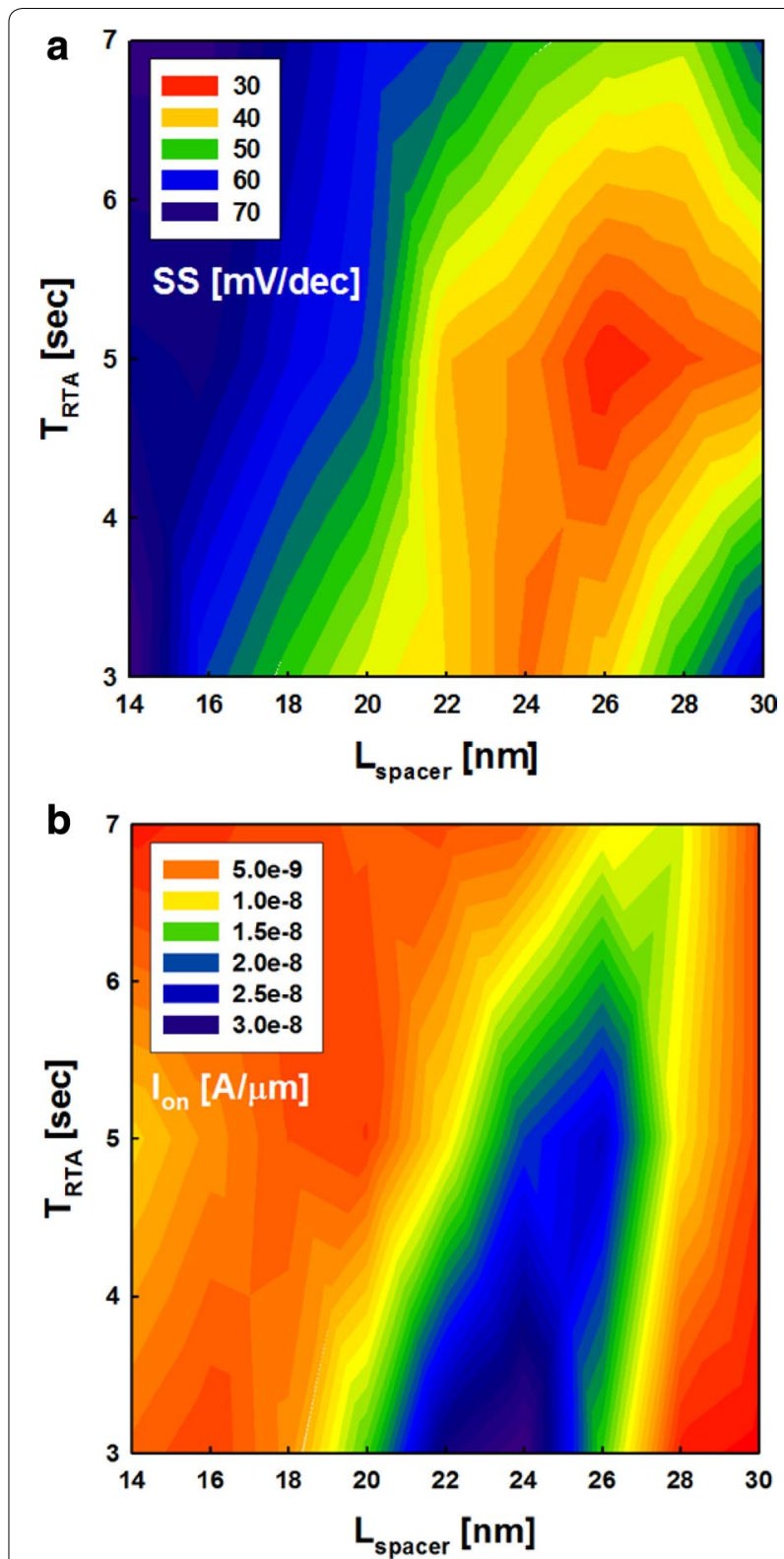

Fig. 13 Contour plots of $\mathbf{a}$ SS and $\mathbf{b}$ I $_{\text {on }}$ for the proposed HG TFETS with the variation of $T_{\text {RTA }}$ and $L_{\text {spacer }}$

strongly influenced by doping profiles. Mostly optimized $I_{\text {on }}$ is shown when $T_{\mathrm{RTA}}$ is $3 \mathrm{~s}$ because more abrupt doping profile is formed as $T_{\mathrm{RTA}}$ decreases. When $T_{\mathrm{RTA}}$ is $3 \mathrm{~s}$, optimum $L_{\text {spacer }}$ is $24 \mathrm{~nm}$ as same as in the case of SS.

From the results of simulation, overlapped region between Fig. 13a, b is selected as the target for the fabrication condition. Finally, optimized $L_{\text {spacer }}$ is $24 \mathrm{~nm}$ and $T_{\mathrm{RTA}}$ is $3 \mathrm{~s}$. Though there is variability from the fabrication conditions, it would be within the margin of error because SS shows little change. 


\section{Fabrication of HG TFETs and analysis}

\subsection{Improvement in fabrication methods}

As discussed in chapter 2, performance degradation was shown for previous HG TFETs and reasons are closely related to fabrication issues. Gradual doping profile is one of them and it is difficult to be improved because it needs advanced annealing equipments. However, there are solutions for enlarged high- $k$ dielectric thickness at the source side and the structure of the sidewall spacer. Two key ideas have been introduced to enhance the performance of HG TFETs in this work.

Figure 14 shows the key process flow to form HG and spacer structure of previous and proposed HG TFETs. In previous work, 7:1 BHF solution was used to etch $\mathrm{SiO}_{2}$ gate insulator. However, this method increased the thickness of the etched $\mathrm{SiO}_{2}$ gate insulator which would be filled with high- $k$ material. Because BHF etched $n^{+}$-doped polysilicon as well as $\mathrm{SiO}_{2}$ gate insulator, corner of the gate was also etched. As a result, thickness of $\mathrm{HfO}_{2}\left(\mathrm{~T}_{\mathrm{HOO}_{2}}\right)$ was larger than thickness of $\mathrm{SiO}_{2}$ layer $\left(T_{\mathrm{SiO}_{2}}\right)$ especially at the edge of the polysilicon gate. This decreased difference of gate-to-channel coupling strength between channel regions overlapped by the high- $k$ material and $\mathrm{SiO}_{2}$ layer which mainly determines the performance of HG TFETs. This problem has been improved by using HF vapor to etch the $\mathrm{SiO}_{2}$ gate insulator at the source side. HF vapor showed much better selectivity compared to 7:1 BHF solution and it enhanced thickness uniformity between $T_{\mathrm{HfO} 2}$ and $T_{\mathrm{SiO} 2}$. While etching the $\mathrm{SiO}_{2}$ insulator, the sample was held at $40{ }^{\circ} \mathrm{C}$. It is because etch rate is too high to control and uniformity is bad when the temperature is lower than $40{ }^{\circ} \mathrm{C}$ and etch rate is too low when the temperature is higher than $40^{\circ} \mathrm{C}$.

Additionally, process for formation of the HG structure has been changed to remove the high- $k$ dielectric layer on the source region. In previous work, outer TEOS spacers were formed right after $\mathrm{HfO}_{2}$ ALD process and then residual $\mathrm{HfO}_{2}$ was removed. As a result, $\mathrm{HfO}_{2}$ layers were remained under TEOS spacers and this decreased energy band of the source region because of increased fringe field from the gate when gate bias is applied [28]. This led to increase of $W_{\text {tun }}$ and degraded performance of HG TFETs. This problem has been improved by etching $\mathrm{HfO}_{2}$ layers before TEOS spacers were formed. In this case, anisotropic $\mathrm{HfO}_{2}$ etching process should be defined to protect the $\mathrm{HfO}_{2}$ layer inserted under the gate. Thus, inductively coupled plasma (ICP) dry etcher was used to etch $\mathrm{HfO}_{2}$ layer on the source region. Adjusting etching time is very important because $\mathrm{HfO}_{2}$ layer on the source region should be removed and $\mathrm{HfO}_{2}$ layer under the gate should be protected at the same time. In addition, very careful control of $\mathrm{HfO}_{2}$ dry etch process was needed because silicon under the $\mathrm{HfO}_{2}$ layerrewis also etched well by $\mathrm{HfO}_{2}$ etch process condition ( $\mathrm{BCl}_{3} 100 \mathrm{sccm}, 700 \mathrm{~W}, 5 \mathrm{~Wb}, 10$ mtorr). As a result, $\mathrm{HfO}_{2}$ layers on the source region were removed and 3-nm inner $\mathrm{HfO}_{2}$ spacers were remained finally.

\subsection{Device fabrication}

In order to fabricate HG TFETs without complexity, the fabrication followed the standard CMOS process. Figure 15 shows key process flow for the fabrication of HG TFETs on SOI wafers. Most of the process steps and device structures are similar to those in previous work [27]. However, performance of fabricated HG TFETs have been improved by changing the method of etching $\mathrm{SiO}_{2}$ layer in Fig. 15d and changing the order of sidewall spacer formation and $\mathrm{HfO}_{2}$ dry etching. P-type (100) 6 -inch SOI wafers $\left(T_{\mathrm{SOI}}=100 \mathrm{~nm}\right.$ and $\left.T_{\mathrm{BOX}}=375 \mathrm{~nm}\right)$ were prepared to reduce the leakage current and $T_{\text {SOI }}$ was reduced to be $30 \mathrm{~nm}$ by thermal oxidation and removing oxide layer. Active patterns were formed on SOI substrate by photolithography and dry etching. Mesa isolation was used to separate each active region by BOX layer. The channel region is doped with p-type at $10^{15} \mathrm{~cm}^{-3}$. By dry oxidation and low-pressure chemical vapor deposition (LPCVD) process, the gate stack of 5-nm-thick $\mathrm{SiO}_{2}$ layer and 100-nm-thick phosphorusdoped polysilicon gate was formed over the active patterned substrate. The most important key process flow of HG TFETs is formation of the HG structure which is divided into two steps. First, $\mathrm{SiO}_{2}$ gate insulator of source side was selectively etched by using HF vapor. Before etching the $\mathrm{SiO}_{2}$ gate insulator only in the source side, photolithography step was performed by using mask for protecting the drain region. Second, atomic layer deposition (ALD) of 5-nm-thick $\mathrm{HfO}_{2}$ was performed to fill the etched gate insulator with high- $k$ material. Then, $\mathrm{HfO}_{2}$ was etched anisotropically to remove the $\mathrm{HfO}_{2}$ on the gate, source and drain regions. Next, sidewall spacer was formed with deposition and etching of TEOS layer. TEOS layer was deposited using PECVD and etched by reactive ion etch (RIE). Next, asymmetric source and doping profiles were obtained by implanting different ions respectively. Compared to MOSFETs which are implemented by self-aligned source and drain ion implantation, two clear field masks for implantation to the source and the drain regions are required as shown in Fig. 16. Each mask for covering source and drain regions during implantation is described with different dotted lines. The mask for implantation to source region is the same as the one which is used when source side $\mathrm{SiO}_{2}$ gate insulator was selectively etched by HF vapor. Mask for implantation to the source region was designed to cover the contact area of the gate region, because gate was doped with n-type and source region was implanted with p-type. Low energy ion implantation was performed for both 


\section{Previous}

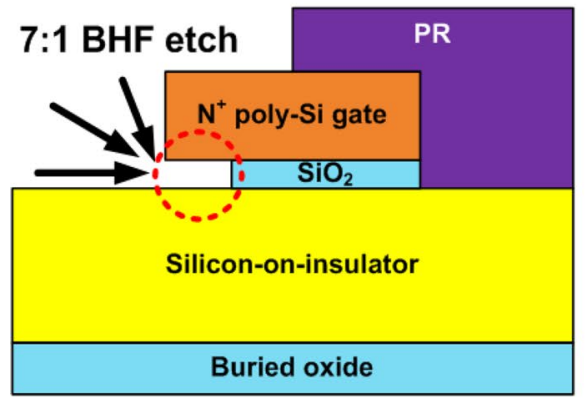

Source-side $\mathrm{SiO}_{2}$ etching by 7:1 BHF

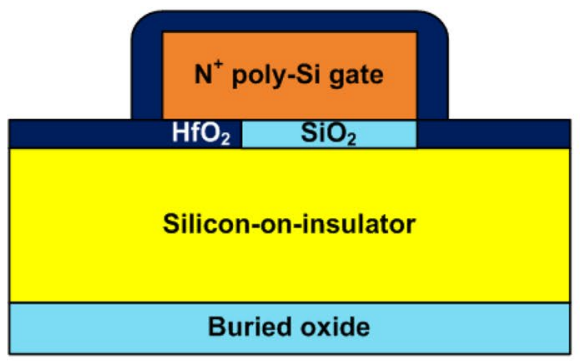

$\mathrm{HfO}_{2}$ atomic layer deposition

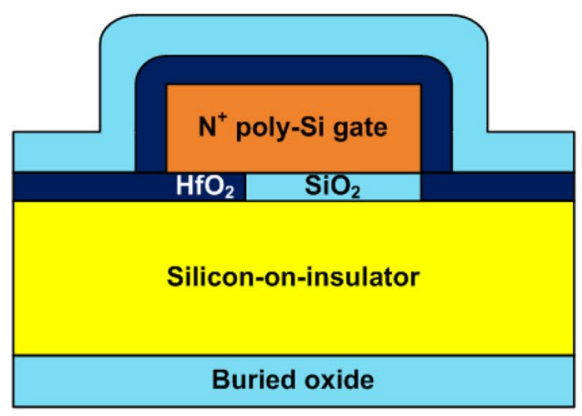

TEOS deposition

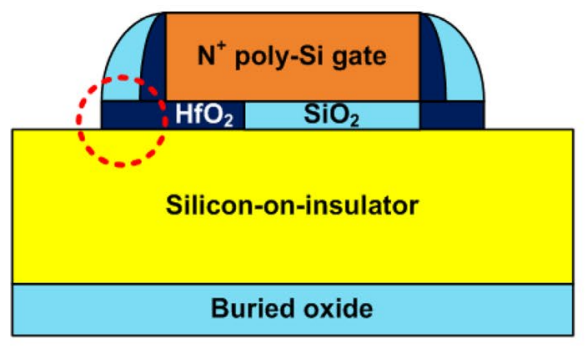

TEOS \& $\mathrm{HfO}_{2}$ etching
Proposed
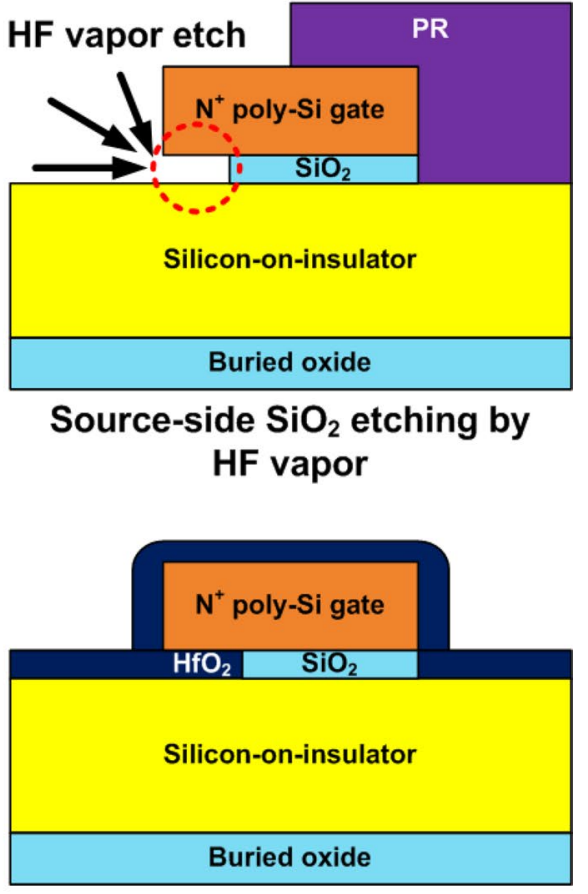

$\mathrm{HfO}_{2}$ atomic layer deposition

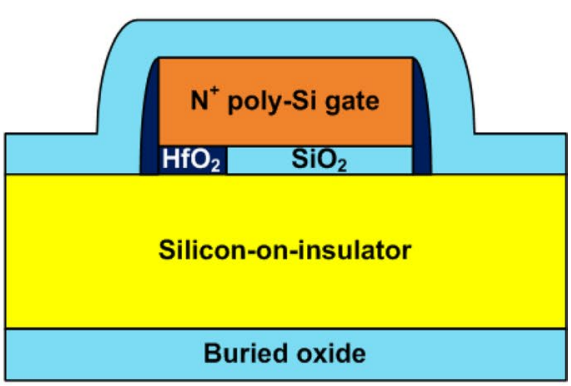

$\mathrm{HfO}_{2}$ etching \& TEOS deposition

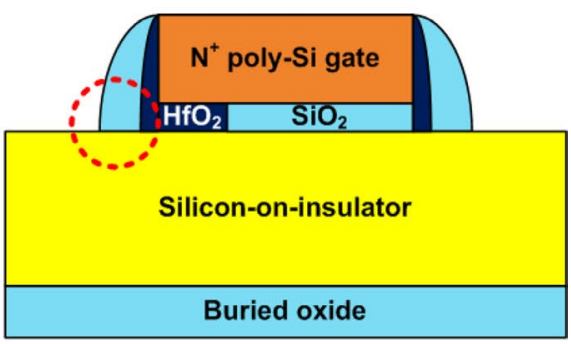

TEOS etching

Fig. 14 Key process proposed for performance improvement of HG TFETs 


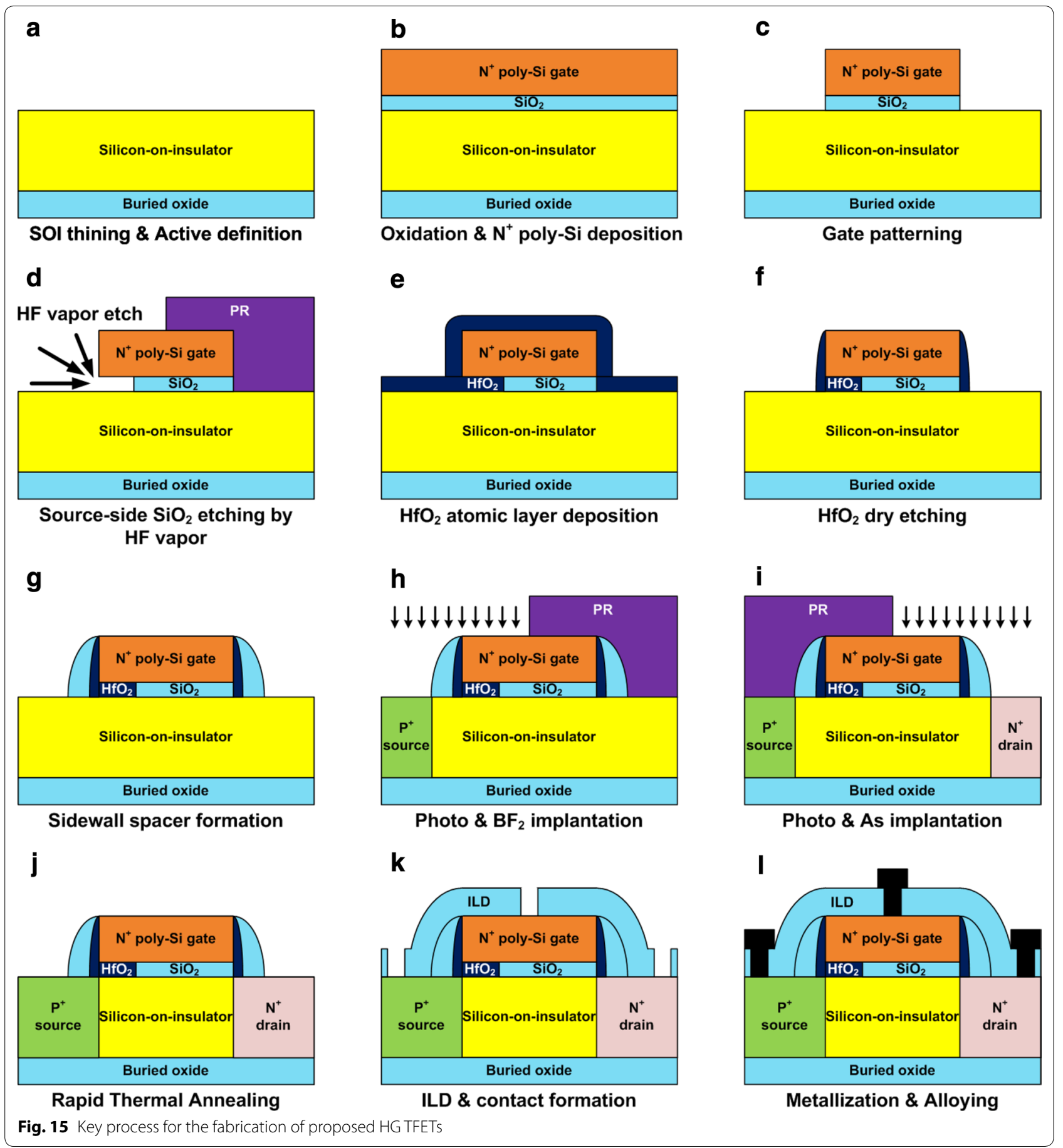

source and drain regions to form a steep junction profile. After photolithography for implanting source region was performed, $\mathrm{BF}_{2}$ ions were implanted with a dose of $1 \times 10^{15} \mathrm{~cm}^{-2}$ at $5 \mathrm{keV}$. Following photoresist stripping, photolithography for implanting drain region was performed and As was implanted with the same condition as implantation for source region. In order to activate the dopants with minimal dopant diffusion, rapid thermal annealing (RTA) was done at $1000{ }^{\circ} \mathrm{C}$ for $3 \mathrm{~s}$. As an interlayer dielectric (ILD) layer, 200-nm-thick TEOS layer was deposited by PECVD which is followed by photolithography for contact hole. Using the photoresist as a mask, ILD was etched down to the gate, source and drain regions by RIE. For a pre metal cleaning, 50:1 
buffered hydrogen fluoride (BHF) solution was used for $30 \mathrm{~s}$. Then, a four-level metallization (Ti-TiN-Al-TiN) was carried out in a sputtering system. Ti was used for metal adhesion, TiN was used for barrier metal, and TiN was used as an antireflection coating for photolithography of $\mathrm{Al}$ metal line. Metal pads were defined by photolithography and etch process. In the final step, forming gas annealing was performed at $450{ }^{\circ} \mathrm{C}$ for $30 \mathrm{~min}$ in $\mathrm{H}_{2} / \mathrm{N}_{2}$ ambient.

Figure 16 shows the top view scanning electron microscope (SEM) image of the fabricated HG TFET. Gate length and width are 1 and $2.7 \mu \mathrm{m}$, respectively. Figure 17 shows the cross-sectional transmission electron microscope (TEM) image of the fabricated HG TFET. $L_{\text {high }-k}$ of the fabricated HG TFET is $\sim 8 \mathrm{~nm}$ which is similar to the optimized value [18]. Additionally, the increase of $T_{\mathrm{HfO}_{2}}$ at the source was improved and $\mathrm{HfO}_{2}$ layer on the source was removed in the proposed HG TFETs as shown in Fig. 17. $T_{\mathrm{HfO}_{2}}$ is almost equal to $T_{\mathrm{SiO}_{2}}$. The spacers consist of 3-nm-wide inner $\mathrm{HfO}_{2}$ spacers and 20-nm-wide outer TEOS spacers. In order to evaluate the merits of HG TFETs, $\mathrm{SiO}_{2}$-only TFETs were also fabricated as control devices. Most of the process flow was the same as that of HG TFETs except for the formation of the HG structure. The $\mathrm{SiO}_{2}$-only TFET has only 20 -nm TEOS spacers.

\subsection{Electrical characteristics}

Figure 18 illustrates the transfer curves of the proposed HG TFETs compared with those of previous HG TFETs

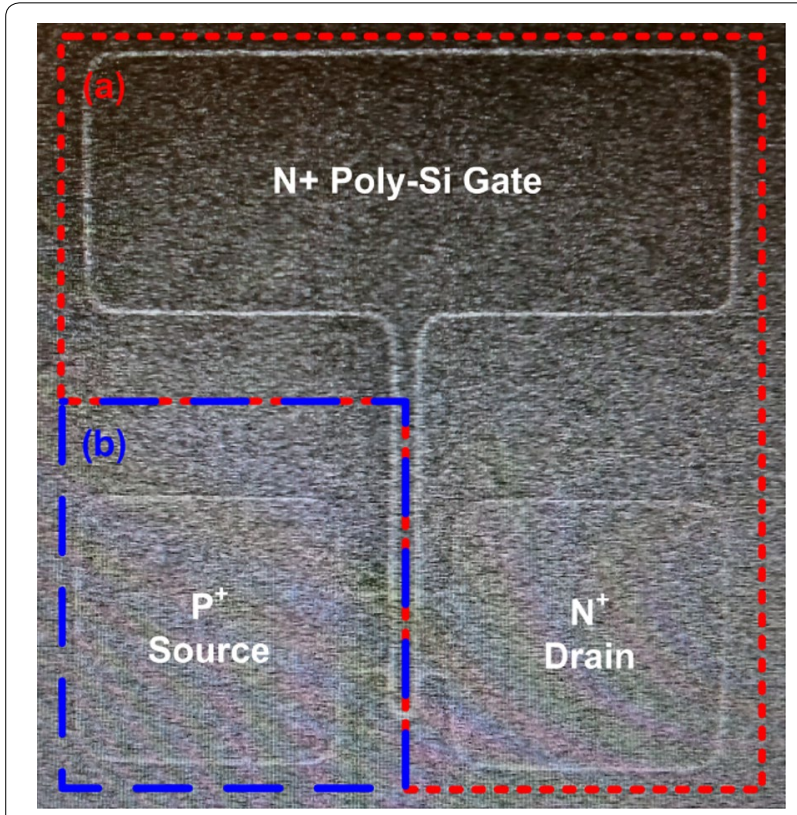

Fig. 16 Top view SEM image of the fabricated HG TFET and masks for implantation to the $\mathbf{a}$ source and $\mathbf{b}$ drain region

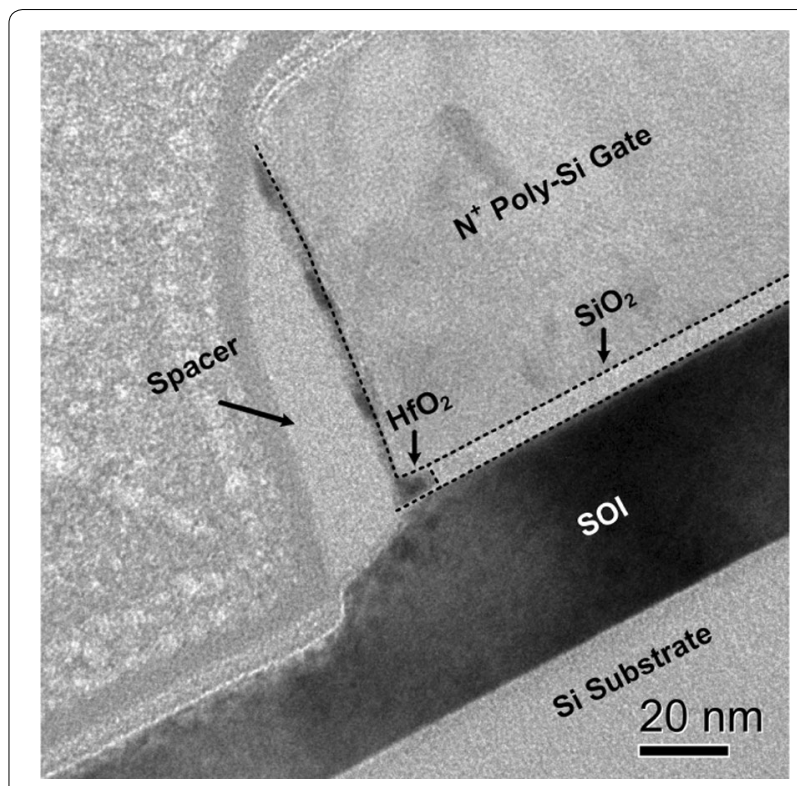

Fig. 17 Cross-sectional TEM image of the fabricated HG TFET

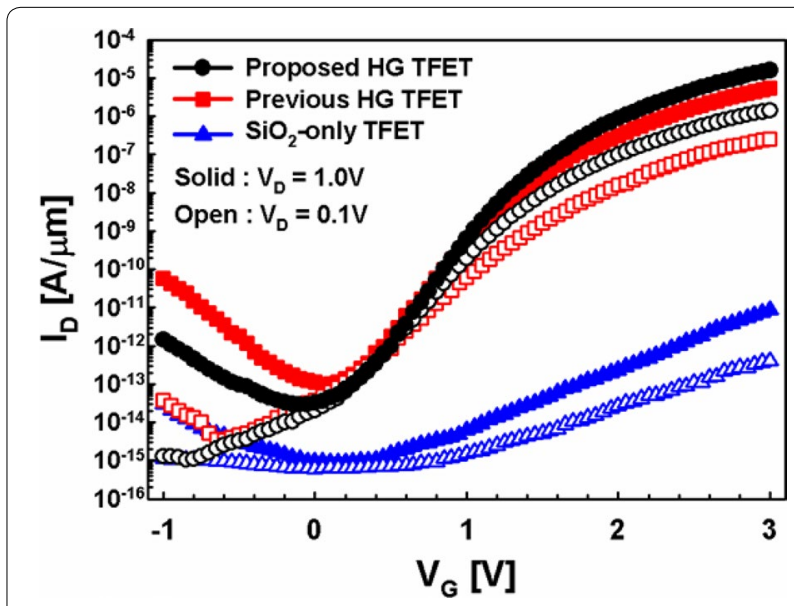

Fig. 18 Transfer characteristics of the proposed and previous HG TFETs and $\mathrm{SiO}_{2}$-only TFETs

and $\mathrm{SiO}_{2}$-only TFETs for $V_{\mathrm{D}}=0.1$ and $1.0 \mathrm{~V}$. Proposed HG TFETs show higher $I_{\text {on }}$ and lower SS than previous HG TFETs as a result of improved device design even though $t_{\mathrm{ox}}$ is $5 \mathrm{~nm}$ which is larger than 3-nm $t_{\mathrm{ox}}$ of previous HG TFETs. In addition, proposed and previous HG TFETs show much higher $I_{\text {on }}$ and lower SS than $\mathrm{SiO}_{2}-$ only TFETs because HG TFETs have a local minimum of $E_{\mathrm{C}}$ resulted from locally inserted $\mathrm{HfO}_{2}$ at the source side gate dielectric. This reduces $W_{\text {tun }}$ and increases the electric field at the tunneling junction. Though both HG and $\mathrm{SiO}_{2}$-only TFETs show low $I_{\text {off }}, I_{\text {amb }}$ of both kinds of devices increases as $V_{\mathrm{D}}$ increases. Especially, $I_{\mathrm{amb}}$ of HG TFETs is larger than that of $\mathrm{SiO}_{2}$-only TFETs because 
inner high- $k$ spacers reduce $W_{\text {tun }}$ between drain and channel region as well as $W_{\text {tun }}$ between source and channel region. Thus, the underlap structure between gate and drain or reducing drain doping concentration are needed to reduce $I_{\text {amb }}[16,17]$.

Figure 19 shows the output characteristics of the proposed and previous HG TFETs. The output characteristics of proposed HG TFETs show better performance and lower parasitic resistance than previous HG TFETs. $I_{\mathrm{D}}$ of both kinds of HG TFETs increases with $V_{\mathrm{D}}$ slowly until it reaches its saturation value at high $V_{\mathrm{D}}$ because of high tunneling resistance. Especially, the tunneling resistance of previous HG TFETs is higher than proposed HG TFETs because $W_{\text {tun }}$ is larger than proposed HG TFETs.

Output characteristics of TFETs are different from those of MOSFETs because their mechanisms are different. While MOSFETs are saturated when the inversion layer of drain side is disappeared, most of inversion layer of TFETs is formed from the drain and surface channel potential $\left(\Psi_{\mathrm{s}}\right)$ is pinned by $V_{\mathrm{D}}$ [30]. Thus, inversion layer formation makes $I_{\mathrm{D}}$ less sensitive to $V_{\mathrm{G}}$ and low $V_{\mathrm{D}}$ results in low $\Psi_{\mathrm{s}}$ which induces band-to-band tunneling currents. However, saturated currents become sensitive to $V_{\mathrm{G}}$ because $I_{\mathrm{D}}$ is determined by band-to-band tunneling without $V_{\mathrm{D}}$ influence when $I_{\mathrm{D}}$ is saturated. Figure 20 shows SS of proposed and previous HG TFETs in terms of $I_{\mathrm{D}}$. SS of $\mathrm{SiO}_{2}$-only TFETs is not considered because SS is much higher than those of both HG TFETs. Proposed HG TFETs show lower SS within wider current range than previous HG TFETs. Table 2 summarizes electrical characteristics of proposed HG TFET compared with those of previous HG TFET and $\mathrm{SiO}_{2}$-only TFET. Dimensions of proposed HG TFET are same as previous HG TFET but $T_{\text {ox }}$ is different at this time. $I_{\text {off }}$ is defined as

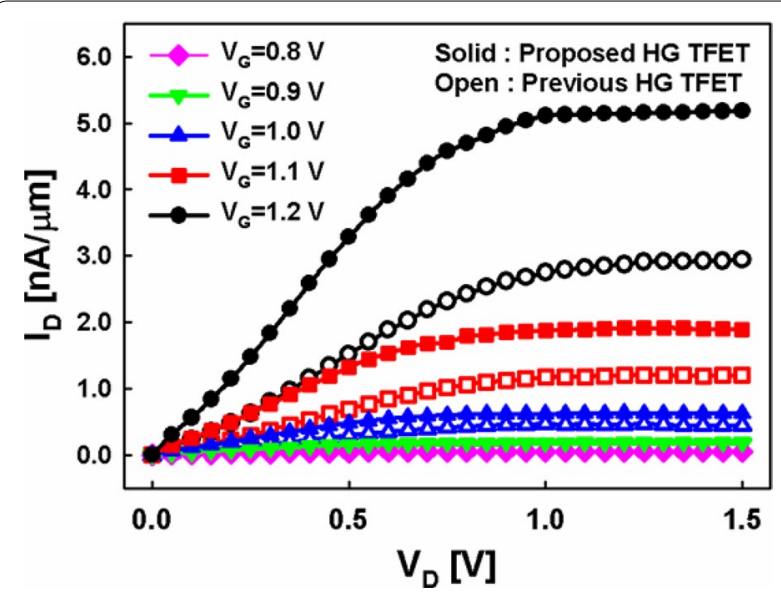

Fig. 19 Output characteristics of the proposed and previous HG TFETS

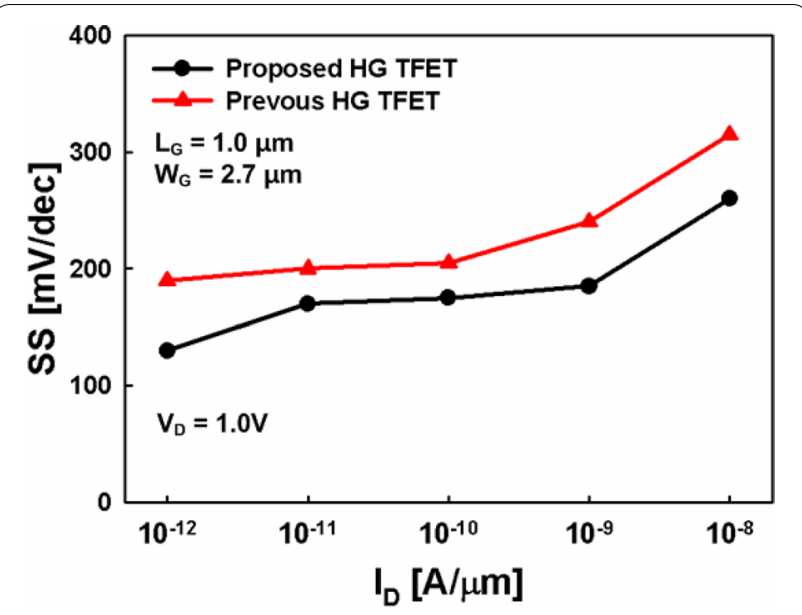

Fig. 20 SS of the proposed and previous HG TFETs in terms of $I_{D}$

Table 2 Electrical characteristics summarization of proposed $\mathrm{HG}$ TFET compared with previous $\mathrm{HG}$ TFET and $\mathrm{SiO}_{2-}$ only TFET

\begin{tabular}{llll}
\hline & $\begin{array}{l}\text { Proposed HG } \\
\text { TFET }\end{array}$ & $\begin{array}{l}\text { Previous HG } \\
\text { TFET }\end{array}$ & SiO $_{2}$-only TFET \\
\hline$L_{G}(\mu \mathrm{m})$ & 1.0 & 1.0 & 1.0 \\
$W_{G}(\mu \mathrm{m})$ & 2.7 & 2.7 & 2.7 \\
$T_{\text {ox }}(\mathrm{nm})$ & 5.0 & 3.0 & 5.0 \\
$V_{\mathrm{DD}}(\mathrm{V})$ & 1.0 & 1.0 & 1.0 \\
$I_{\text {on }}(\mathrm{nA} / \mu \mathrm{m})$ & 56 & 18 & 0.004 \\
$I_{\text {min }}(\mathrm{pA} / \mu \mathrm{m})$ & 0.03 & 0.1 & 0.001 \\
$\mathrm{SS}_{\text {min }}(\mathrm{mV} / \mathrm{dec})$ & 130 & 170 & 550 \\
$\mathrm{SS}_{\text {avg }}(\mathrm{mV} / \mathrm{dec})$ & 170 & 200 & 650 \\
$I_{\text {on }} / I_{\text {off }}$ & $5.6 \times 10^{4}$ & $1.8 \times 10^{4}$ & $3.5 \times 10$ \\
\hline & & &
\end{tabular}

$I_{\mathrm{D}}$ is equal to $1 \mathrm{pA} / \mu \mathrm{m}$ and $V_{\text {off }}$ is defined as the $V_{\mathrm{G}}$ when $I_{\mathrm{D}}$ is $I_{\text {off }} I_{\text {on }}$ is defined as $I_{\mathrm{D}}$ when $V_{\mathrm{G}}$ is $V_{\text {off }}+V_{\mathrm{DD}} \cdot \mathrm{SS}_{\text {min }}$ is minimum point swing and $\mathrm{SS}_{\mathrm{avg}}$ is an average slope when $I_{\mathrm{D}}$ is from $1 \mathrm{pA} / \mu \mathrm{m}$ to $1 \mathrm{nA} / \mu \mathrm{m}$. Proposed HG TFET shows higher $I_{\text {on }}$ and lower $I_{\min }$ than previous HG TFET even though $T_{\text {ox }}$ is increased. $\mathrm{SS}_{\min }$ and $\mathrm{SS}_{\text {avg }}$ of proposed HG TFET are also lower than those of previous HG TFET. In addition, proposed HG TFET has an $I_{\text {on }} / I_{\text {off }}$ of $5.6 \times 10^{4}$ at $V_{\mathrm{DD}}=1 \mathrm{~V}$ which is comparable with other reported Si TFETs [8, 11, 12, 22].

Although HG TFETs are proposed for low-power application, SS of HG TFETs is larger than $60 \mathrm{mV} / \mathrm{dec}$ and current drivability is much smaller than requirements of the Low Standby Power devices [31]. First of all, abrupt doping profile is necessary for higher $I_{\mathrm{on}}$ and lower SS. Because conventional RTA is used in this work, $W_{\text {tun }}$ is increased and it is difficult to control the tunneling junction. Advanced annealing methods such as spike or 
laser annealing can be considered for this purpose [23, 32]. In addition, tunneling current can be enhanced by using lower bandgap semiconductors such as $\mathrm{SiGe}, \mathrm{Ge}$ and III-V materials [17, 19-21]. If relative permittivity of high- $k$ material increases, performance of HG TFETs would be further improved.

\section{Conclusions}

In this work, HG TFETs have been investigated through the simulation and fabrication of devices in order to demonstrate the higher performance and low-power consumption. Optimized HG TFETs showed higher $I_{\text {on }}$ and, lower $I_{\mathrm{amb}}$ and SS than conventional TFETs by replacing source-side gate insulator with a high- $k$ material. A high$k$ material partially located at the source side induced a local minimum of $E_{\mathrm{c}}$ due to relative permittivity discrepancy between high- $k$ dielectric and $\mathrm{SiO}_{2}$ layer. In addition, proposed HG TFETs showed improved device performance than previous HG TFETs by improvement in device design. For the fabrication of HG TFETs with improved performance, key processes were modified. HF vapor was used to etch the source-side gate insulator uniformly and $\mathrm{HfO}_{2}$ etch was performed right after $\mathrm{HfO}_{2}$ ALD to remove the $\mathrm{HfO}_{2}$ layer remained on the source region. Through the electrical test of fabricated devices, proposed HG TFETs showed higher performance than previous HG TFETs and conventional TFETs in terms of $I_{\text {on }}$ and SS. To sum up, it is promising that HG TFETs are alternative devices which will complement the MOSFETs for highly energy efficient ICs.

\section{Authors' contributions}

All authors have contributed to the writing of the manuscript. Both authors read and approved the final manuscript.

\section{Acknowledgements \\ This work was supported in part by the NRF of Korea funded by the MSIP under Grant NRF-2015003565 (Mid-Career Researcher Program), NRF- 2015046617 (Fundamental Technology Program) and in part by the MOTIE/ KSRC under Grant 10044842 (Future Semiconductor Device Technology Development Program).}

\section{Competing interests}

The authors declare that they have no competing interests.

Received: 9 March 2016 Accepted: 2 May 2016

Published online: 15 June 2016

\section{References}

1. M. Bohr, The new era of scaling in an SoC world, in IEEE International SolidState Circuits Conference (ISSCC) (2009), pp. 23-28

2. D.J. Frank, R.H. Dennard, E. Nowak, P.M. Solomon, Y. Taur, H.-S.P. Wong, Device scaling limits of Si MOSFETs and their application dependencies. Proc. IEEE 89(3), 259-288 (2001)

3. L. Chang, Y.-K. Choi, D. Ha, P. Ranade, S. Xiong, J. Bokor, C. Hu, T.-J, King, Extremely scaled silicon nano-CMOS devices. Proc. IEEE 91(11), 1860-1873 (2003)
4. Y. Taur, E. Nowak, CMOS devices below $0.1 \mu \mathrm{m}$ : how high will performance go? in IEEE International Electron Devices Meeting (IEDM) Technical Digest (1997), pp. 215-218

5. K. Gopalakrishnan, P. B. Griffin, J. D. Plummer, I-MOS: a novel semiconductor device with a subthreshold slope lower than $\mathrm{kT} / \mathrm{q}$, in IEEE International Electron Devices Meeting (IEDM) Technical Digest (2002), pp. 289-292

6. W.Y. Choi, J.Y. Song, J.D. Lee, Y.J. Park, B.-G. Park, 100-nm n-/p-channel I-MOS using a novel self-aligned structure. IEEE Electron Device Lett. 26(4), 261-263 (2005)

7. H. Kam, D.T. Lee, R.T. Howe, T.J. King, A new nano-electromechanical field effect transistor (NEMFET) design for low-power electronics, in IEEE International Electron Devices Meeting (IEDM) Technical Digest (2005), pp. 463-466

8. P.-F. Wang, K. Hilsenbeck, Th Nirschl, M. Oswald, C. Stepper, M. Weiss, D. Schmitt-Landsiedel, W. Hansch, Complementary tunneling transistor for low power application. Solid-State Electron. 48(12), 2281-2286 (2004)

9. T. Nirschl, P.-F. Wang, C. Weber, J. Sedlmeir, R. Heinrich, R. Kakoshke, K. Schrufer, J. Holz, C. Pacha, T. Schulz, M. Ostermayr, A. Olbrich, G. Georgakos, E. Ruderer, W. Hansch, D. Schmitt-Landsiedel, The tunneling field effect transistor (TFET) as an add-on for ultra-low-voltage analog and digital processes, in IEEE International Electron Devices Meeting (IEDM) Technical Digest, (2004), pp. 195-198

10. Q. Zhang, W. Zhao, A. Seabaugh, Low-subthreshold-swing tunnel transistors. IEEE Electron Device Lett. 27(4), 297-300 (2006)

11. W.Y. Choi, B.-G. Park, J.D. Lee, T.-J.K. Liu, Tunneling field-effect transistors (TFETs) with subthreshold swing (SS) less than $60 \mathrm{mV} / \mathrm{dec}$. IEEE Electron Device Lett. 28(8), 743-745 (2007)

12. R. Gandhi, Z. Chen, N. Singh, K. Banerjee, S. Lee, Vertical Si-nanowire n-type tunneling FETs with low subthreshold swing ( $\leq 50 \mathrm{mV} /$ decade) at room temperature. IEEE Electron Device Lett. 32(4), 437-439 (2011)

13. J. Appenzeller, Y.-M. Lin, J. Knoch, P. Avouris, Band-to-band tunneling in carbon nanotube field-effect transistors. Phys. Rev. Lett. 93(19), 196805 (2004)

14. K. Boucart, A.M. lonescu, Length scaling of the double gate tunnel FET with a high-k dielectric. Solid-State Electron. 51(11-12), 1500-1507 (2007)

15. J. Knoch, S. Mantl, J. Appenzeller, Impact of the dimensionality on the performance of tunneling FETs: bulk versus one-dimensional devices. Solid-State Electron. 51(4), 572-578 (2007)

16. J.S. Jang, W.Y. Choi, Ambipolarity factor of tunneling field-effect transistors (TFETs). J. Semicond. Technol. Sci. 11(4), 272-277 (2011)

17. T. Krishnamohan, D. Kim, S. Raghunathan, K. Saraswat, Double gate strained-Ge heterostructure tunneling FET (TFET) with record high drive currents and $<60 \mathrm{mV} /$ dec subthreshold slope, in IEEE International Electron Devices Meeting (IEDM) Techical Digest (2008), pp. 947-949

18. W.Y. Choi, W. Lee, Hetero-gate-dielectric tunneling field-effect transistors. IEEE Trans. Electron Devices 57(9), 2317-2319 (2010)

19. S.H. Kim, H. Kam, C. Hu, T.-J. K. Liu, Germanium-source tunnel field effect transistors with record high I ON/ OFF, $_{\text {, }}$ in VLSI Symposium Technical Digest, 2009 (2009), pp. 178-179

20. G. Zhou, Y. Lu, R. Li, Q. Zhang, W.S. Hwang, Q. Liu, T. Vasen, C. Chen, H. Zhu, J.-M. Kuo, S. Koswatta, T. Kosel, M. Wistey, P. Fay, A. Seabaugh, H. Xing, Vertical InGaAs/InP tunnel FETs with tunneling normal to the gate. IEEE Electron Device Lett. 32(11), 1516-1518 (2011)

21. K. E. Moselund, H. Ghoneim, M. T. Bjork, H. Schmid, S. Karg, E. Lortscher, W. Riess, H. Riel, Comparison of VLS grown Si NW tunnel FETs with different gate stacks, in Proceedings of the European Solid-State Device Research Conference (ESSDERC) (2009), pp. 448-451

22. F. Mayer, C. Le Royer, J.-F. Damlencourt, K. Romanjek, F. Andrieu, C. Tabone, B. Previtali, S. Deleonibus, Impact of $\mathrm{SOI}, \mathrm{Si}_{1-x} \mathrm{Ge}_{x} \mathrm{Ol}$ and $\mathrm{GeOl}$ substrates on CMOS compatible tunnel FET performance, in IEEE International Electron Devices Meeting (IEDM) Technical Digest (2008), pp. 163-166

23. R. Jhaveri, V. Nagavarapu, J.C.S. Woo, Effect of pocket doping and annealing schemes on the source-pocket tunnel field-effect transistor. IEEE Trans. Electron Devices 58(1), 80-86 (2011)

24. SILVACO International, Santa Clara, CA 95054, USA, ATHENA/ATLAS User's Manual (2012)

25. G. Lee, W.Y. Choi, Low-power circuit applicability of hetero-gate-dielectric tunneling field-effect transistors. IEICE Trans. Electron. E95-C(5), 910-913 (2012) 
26. M.J. Lee, W.Y. Choi, Effect of device geometry on hetero-gate-dielectric tunneling field-effect transistors (HG TFETs). IEEE Electron Device Lett. 33(10), 1459-1461 (2012)

27. G. Lee, W.Y. Choi, Dual-dielectric-constant spacer hetero-gate-dielectric tunneling field-effect transistors. Semicond. Sci. Technol. 28, 052001 (2013)

28. H.G. Virani, R.B.R. Adari, A. Kottantharayil, Dual-k spacer device architecture for the improvement of performance of Silicon n-channel tunnel TFETs. IEEE Trans. Electron Devices 57(10), 2410-2417 (2010)

29. A. Chattopadhyay, A. Mallik, Impact of a spacer dielectric and a gate overlap/underlap on the device performance of a tunnel field-effect transistors. IEEE Trans. Electron Devices 58(3), 677-683 (2011)
30. W. Lee, W.Y. Choi, Influence of inversion layer on tunneling field-effect transistors. IEEE Electron Device Lett. 32(9), 1191-1193 (2011)

31. The International Technology Roadmap for Semiconductors (ITRS) (2012), http://www.itrs2.net/. Accessed 6 Jan 2016

32. D. Leonelli, A. Vandooren, R. Rooyackers, S.D. Gendt, M.M. Heyns, G. Groeseneken, Drive current enhancement in p-tunnel FETs by optimization of the process conditions. Solid-State Electron. 65-66, 28-32 (2011)

\section{Submit your manuscript to a SpringerOpen ${ }^{\circ}$ journal and benefit from:}

- Convenient online submission

\section{- Rigorous peer review}

- Immediate publication on acceptance

- Open access: articles freely available online

- High visibility within the field

- Retaining the copyright to your article 